\title{
OPTIMAL CONTROL OF A THERMISTOR PROBLEM WITH VANISHING CONDUCTIVITY
}

\author{
VOLODYMYR HRYNKIV* AND SERGIY KOSHKIN ${ }^{\dagger}$
}

\begin{abstract}
An optimal control of a steady state thermistor problem is considered, where the convective boundary coefficient is taken as the control variable. A distinctive feature of this paper is that the problem is considered in arbitrary dimensions, and the electrical conductivity is allowed to vanish above a threshold temperature value. The existence of a steady state is proved, an objective functional is introduced, the existence of the optimal control is proved, and the optimality system is derived.
\end{abstract}

Key words. optimal control, thermistor problem, elliptic systems

AMS subject classifications. 49J20, 49K20

1. Introduction. Thermistor is a device whose electrical conductivity is highly sensitive to temperature, namely, its electrical conductivity may change by several orders of magnitude with the increase of temperature. Thermistors are often used as temperature control elements in a wide variety of military and industrial equipment ranging from space vehicles to air conditioning controllers. They are also used in the medical field for localized and general body temperature measurement, in meteorology for weather forecasting, and in chemical industries as process temperature sensors [15, 17. A detailed description of thermistors and their applications in electronics and other industries can be found in [17.

We consider the following steady-state thermistor problem

$$
\begin{aligned}
\nabla \cdot(\sigma(u) \nabla \varphi) & =0 & & \text { in } \Omega, \\
\Delta u+\sigma(u)|\nabla \varphi|^{2} & =0 & & \text { in } \Omega, \\
\frac{\partial u}{\partial n}+\beta\left(u-u_{1}\right) & =0 & & \text { on } \Gamma_{R}, \\
u & =u_{0} & & \text { on } \Gamma_{D}, \\
\varphi & =\varphi_{0} & & \text { on } \partial \Omega,
\end{aligned}
$$

where $\varphi(x)$ is the electric potential, $u(x)$ is the temperature, $\sigma(u)$ is the electrical conductivity, and $n$ denotes the outward unit normal. A more detailed discussion of the physical justification of equations (1.1) can be found in [10,13, 21].

The heat transfer coefficient $\beta$ is taken to be a control variable and the set of admissible controls is denoted by $U_{\mathcal{M}}:=\left\{\beta \in L^{\infty}\left(\Gamma_{R}\right): 0 \leq \beta \leq \mathcal{M}\right\}$. Assumptions on the data are as follows:

1. $\Omega \subset \mathbb{R}^{d}, d>2$, is a bounded domain with piecewise smooth (at least $C^{2}$ ) boundary $\partial \Omega$, where $\partial \Omega=\bar{\Gamma}_{D} \cup \bar{\Gamma}_{R}$ with $\Gamma_{D} \neq \emptyset$.

2. $\sigma(s) \in C^{1}([0, \infty)), \sigma(s)$ is a positive function, monotone decreasing on $\left[0, u_{*}\right)$, where $u_{*}$ is a threshold temperature, that is, $\sigma(u)=0$ for $u \geq u_{*}$ and $\sigma(u)>0$ for $u<u_{*}$. In addition, $\|\sigma\|_{C^{1}\left(\left[0, u_{*}\right]\right)} \leq \mu<\infty$ and $\int_{0}^{u_{*}} \frac{d s}{\sigma(s)}=\infty$. The value $u_{*}$ is called the critical temperature, where we allow $u_{*}=\infty$, but in realistic thermistors $u_{*}<\infty$.

\footnotetext{
${ }^{*}$ Department of Mathematics and Statistics, University of Houston - Downtown, Houston, TX 77002 USA (HrynkivV@uhd.edu).

${ }^{\dagger}$ Department of Mathematics and Statistics, University of Houston - Downtown, Houston, TX 77002 USA (koshkins@uhd.edu).
} 
3. Extending $\varphi_{0}$ to the whole domain $\Omega$ we assume that $\varphi_{0} \in C^{1}(\bar{\Omega})$, moreover $\left\|\varphi_{0}\right\|_{W^{1, \infty}(\Omega)}$ is sufficiently small.

4. $u_{0}, u_{1} \geq 0$ a.e., $\beta \in U_{\mathcal{M}}$, and also extending to the whole domain $u_{0}, u_{1} \in$ $C^{1}(\bar{\Omega})$. Moreover, $\left\|u_{0}\right\|_{L^{\infty}(\Omega)},\left\|u_{1}\right\|_{L^{\infty}(\Omega)}<u_{*}$, i.e. the boundary data is bounded away from the critical temperature.

The first paper on an optimal control problem for thermistor was a time dependent one considered by Lee and Shilkin in [16, where the source term was taken to be the control. The most recent paper on optimal control of thermistor equations is by Meinlschmidt, Meyer, and Rehberg [18, where the time dependent problem from [12] is extended to three dimensions and where the control is taken to be the current on a part of the boundary. Ammi and Torres in [1] considered an optimal control of nonlocal thermistor equations.

Our paper is a generalization of [14, and to our knowledge this is the first paper on optimal control of thermistor with vanishing conductivity. We generalize 14 in several ways. First of all, as physics demands the electrical conductivity $\sigma(u)$ is no longer assumed to be uniformly positive. In real thermistors the conductivity drops sharply by several orders of magnitude at some critical temperature, and remains essentially zero for larger temperatures, this feature is essential for the intended functioning of thermistors as thermoelectric switches. The restriction to two-dimensional domains is also removed, as well as the artificial strict positivity assumption on the boundary heat transfer coefficient $\beta$ that serves as the control.

The price for removing these restrictions is a somewhat higher regularity imposed on the equilibrium temperature in the Robin condition, and introduction of the Dirichlet boundary condition for the temperature on part of the boundary. Let us explain the reasons.

The proof of existence of optimal control in 14] was based on the existence result of [13, which assumed $\sigma(u)$ to be uniformly positive. Without this assumption the first equation in (1.1) is not uniformly elliptic, so there may be no a priori bound on $\nabla \varphi$, and $\sigma(u) \nabla \varphi$ may not make sense. One way to deal with this issue is to switch to a notion of the capacity solution developed by Xu 23 25. Unfortunately, his theory is restricted to the Dirichlet/Neumann boundary conditions, and does not provide enough regularity to construct the optimal control.

We adopt instead the approach developed by Chen [5] for the thermistor problem with the Robin condition on part of the boundary (actually, Chen's condition is even more general). It uses a modified Diesselhörst substitution to transform (1.1) into a similar problem where the analog of $\sigma(u)$ no longer vanishes. The boundary condition becomes non-linear, and the resulting equation for the analog of $u$ is "not very good", as Chen put it (one has to work with a differential inequality), but after some technical labor one is able to derive $L^{\infty}$ bounds on $u$ that bound it away from the critical temperature. The Chen's original work states the assumptions about the data only in terms of the transformed problem, and focuses on the assumptions that do not obtain for $\sigma(u)$ of interest to us. So our contribution in this part is to spell out the assumptions on the original data (using Lemma 2.3), and to somewhat rework the proof.

To reap the fruits of our labor, however, we need a higher regularity for $u$ than $L^{\infty}$, so that we can get $\varphi$ which is better than $H^{1}$ for constructing the control. The requisite improvement to $W^{1, p}$, with any $p>2$, based on the first equation in (1.1), is provided by Theorem 7.2(iii) of [20, but only assuming that $u$ (and hence $\sigma(u)$ ) is in $C^{0}$. Chen's paper does provide the bootstrap to Hölder continuity for $u$ (and $\varphi$ ) 
once the $L^{\infty}$ bound is in place, but only if $C^{1}$ regularity is assumed of the boundary data. The restriction to two dimensions is removed because we no longer depend on the Meyers estimate relied upon by [13] to handle the boundary data with weaker regularity. We believe that to accomodate discontinuous boundary data one would have to extend Xu's capacity solutions to the Robin problem, and develop non-smooth methods for constructing controls in this context.

In [14] the Robin condition on $u$ was imposed along the entire boundary, and the heat transfer coefficient $\beta$, which serves as the control, was bounded away from zero. Unfortunately, this does not provide enough coercivity to guarantee that $u$ stays away from the critical temperature. To keep it from going critical one needs to know explicitly that $u<u_{*}$ at least somewhere in the domain, this is the reason for imposing the Dirichlet condition on part of the boundary with $\left\|u_{0}\right\|_{L^{\infty}(\Omega)}<u_{*}$. Once this is done, however, the uniform positivity of $\beta$ is no longer necessary on the Robin part of the boundary.

In this paper we choose the same objective functional as in [14]. The physical considerations leading to this objective functional can be found in [14] as well. Thus we have

$$
J(\beta)=\int_{\Omega} u d x+\int_{\Gamma_{R}} \beta^{2} d s,
$$

and the optimal control problem is:

$$
\text { Find } \beta^{*} \in U_{\mathcal{M}} \text { such that } J\left(\beta^{*}\right)=\min _{\beta \in U_{\mathcal{M}}} J(\beta) \text {. }
$$

Henceforth we use the standard notation for Sobolev spaces, we denote $\|\cdot\|_{p}=\|\cdot\|_{L^{p}(\Omega)}$ for each $p \in[1, \infty]$; other norms will be explicitly labeled.

2. Existence of a weak solution and its regularity. First, let us define weak solution to (1.1). Denote $V_{D}:=\left\{v \in H^{1}(\Omega): v=0\right.$ on $\left.\Gamma_{D}\right\}$. A pair $(u, \varphi)$ is called a weak solution if $u-u_{0} \in V_{D}, \varphi-\varphi_{0} \in H_{0}^{1}(\Omega)$, and

$$
\begin{gathered}
\int_{\Omega} \nabla u \nabla v d x+\int_{\Gamma_{R}} \beta\left(u-u_{1}\right) v d s=\int_{\Omega}\left(\varphi_{0}-\varphi\right) \sigma(u) \nabla \varphi \nabla v d x+\int_{\Omega}\left(\sigma(u) \nabla \varphi \nabla \varphi_{0}\right) v d x \\
\int_{\Omega} \sigma(u) \nabla \varphi \cdot \nabla w d x=0 \quad \forall w \in H_{0}^{1}(\Omega), \forall v \in V_{D}(\Omega) .
\end{gathered}
$$

The key existence result that we rely on is the following, the proof will be given in the next two sections.

TheOREm 2.1. Suppose conditions 1.-4. of the Introduction hold. Then system (1.1) has a weak solution $u, \varphi$. Moreover, $u, \varphi \in C^{\alpha}(\bar{\Omega})$ for some $0<\alpha<1$ and $0 \leq u \leq N<u_{*}$, where $N$ depends only on $\Gamma_{D}, u_{0}, \varphi_{0}$ and $\|\sigma\|_{C^{1}}$, but not on $u_{1}$ or $\beta$.

For the proof of the existence of optimal control we will need a better regularity for $\varphi$ than simply $H^{1}$, which follows from the following result (see [20], Theorem 7.2(iii), p. 82).

THEOREM 2.2. Let $\Omega \subset \mathbb{R}^{d}$ be a bounded domain with $\partial \Omega \in C^{1}$ and suppose that $u \in H_{0}^{1}(\Omega)$ is the unique solution to

$$
\int_{\Omega}\left(a_{i j} \phi_{x_{i}}+f_{j}\right) v_{x_{j}} d x=0, \forall v \in H_{0}^{1}(\Omega)
$$


where $a_{i j} \in C^{0}(\bar{\Omega})$ ) and strictly elliptic. Then $\phi \in W_{0}^{1, r}(\Omega)$ for each $2<r<\infty$, whenever $f_{j} \in L^{r}(\Omega), j=1, \ldots, n$.

Applying this theorem to the first equation in (1.1) rewritten for $\varphi-\varphi_{0}$ we see that $a_{i j}=\sigma \circ u$ is even in $C^{\alpha}(\bar{\Omega})$, and strictly positive because $u<u_{*}$, while $f_{j}=(\sigma \circ u) \nabla \varphi_{0}$ is even in $C^{0}(\bar{\Omega})$. Therefore, Theorem 2.2 guarantees that $\varphi-\varphi_{0} \in W_{0}^{1, r}(\Omega)$, and hence $\varphi \in W^{1, r}(\Omega)$, for each $2<r<\infty$.

Thus, we can always choose $r$ and then $s$ in such a way that

$$
\begin{gathered}
r>d>2, s \in\left[1, \frac{2 d}{d-2}\right), \text { and } \\
\frac{1}{s}+\frac{1}{r}=\frac{1}{2} .
\end{gathered}
$$

Namely, given $d>2$ choose $r=2(d-1)$ and $s=2(d-1) /(d-2)$. Observe that this choice of $s$ guarantees that $s \in\left[1, \frac{2 d}{d-2}\right)$ and hence $H^{1}(\Omega) \subset \subset L^{s}(\Omega)$ for all $s \in\left[1, \frac{2 d}{d-2}\right)$. On the other hand, the selection of $r>d$ will guarantee the compact embedding $W^{1, r}(\Omega) \subset \subset C(\bar{\Omega})$. These (compact) embeddings will be used when both existence of optimal control and derivation of optimality system are considered.

2.1. Diesselhorst-Chen substitution. The proof of Theorem 2.1 relies on analyzing a system obtained from (1.1) by a modification of a well-known substitution. The usual Diesselhorst substitution [9] is $\tilde{\psi}=\frac{1}{2} \varphi^{2}+F(u)$, where $F(u):=\int_{0}^{u} \frac{d s}{\sigma(u)}$. We will use a modified substitution, introduced by Chen [5], $\psi:=\left(\varphi-\varphi_{0}\right)^{2}+F(u)$ to accommodate the Robin boundary condition. First, note that $F$ maps $\left[0, u_{*}\right)$ onto $[0, \infty)$ since $\int_{0}^{u} \frac{d s}{\sigma(s)}=\infty$ by assumption, and $F^{-1}:[0, \infty) \rightarrow[0, u *)$ is well defined. Let $v:=\int_{0}^{u} \frac{d s}{\sigma(s)}=F(u)$, not to be confused with $v$ in (2.1), which was just a generic test function. We introduce $a(v):=\sigma\left(F^{-1}(v)\right)$, and hence $\nabla v=F^{\prime}(u) \nabla u=$ $\frac{1}{\sigma(u)} \nabla u=\frac{1}{\sigma\left(F^{-1}(v)\right)} \nabla u=\frac{1}{a(v)} \nabla u$. Therefore $\nabla u=a(v) \nabla v$, and the original system (1.1) can be written as:

$$
\begin{aligned}
\nabla \cdot(a(v) \nabla \varphi) & =0 \quad \text { in } \Omega, \\
\nabla \cdot(a(v) \nabla v)+a(v)|\nabla \varphi|^{2} & =0 \quad \text { in } \Omega, \\
\frac{\partial v}{\partial n}+\frac{\beta}{a(v)}\left(F^{-1}(v)-u_{1}\right) & =0 \quad \text { on } \Gamma_{R}, \\
v & =F\left(u_{0}\right) \quad \text { on } \Gamma_{D}, \\
\varphi & =\varphi_{0} \quad \text { on } \partial \Omega .
\end{aligned}
$$

As with the system (1.1) we understand (2.3) in the weak sense analogous to (2.1). One advantage of this new system is that now $a(v)>0$ on $[0, \infty)$, unlike $\sigma(u)$. We can now outline the proof of Theorem 2.1 modulo the a priori $L^{\infty}$ estimate of Theorem 2.9. whose very technical proof is postponed until the next section.

Proof of Theorem 2.1. By Theorem 2.9 any weak solution to (2.3) is in $L^{\infty}$, which means that the corresponding weak solution to (1.1) is bounded away from the critical temperature:

$$
0 \leq u \leq F^{-1}\left(\|v\|_{\infty}\right)=: M<u_{*} .
$$

Let $\sigma_{n}(s)$ be equal to $\sigma(s)$ for $s \leq n$, satisfy $\sigma_{n}(s) \geq \sigma(n) / 2$ for all $s \geq 0$, and $\left\|\sigma_{n}\right\|_{C^{1}}=\|\sigma\|_{C^{1}}$. Such a $\sigma_{n}$ can always be produced by interpolation. 
By (2.9) and (2.10) the $L^{\infty}$ estimate for $\|v\|_{\infty}$, and hence the value of $N$, only depend on $\|\sigma\|_{C^{1}}=\mu$ and $u_{0}, \varphi_{0}$. In particular, it is independent of $\beta$, and of $n$. But if $n<u_{*}$ then $\sigma_{n}$ is bounded away from 0 for all $s \geq 0$, so by the main result of [13] system (1.1) with $\sigma$ replaced by $\sigma_{n}$ has a weak solution. For $n>N$ this weak solution will actually be a solution to (1.1) with $\sigma$ itself, and the corresponding $v, \varphi$ will be a weak solution to (2.3) bounded in $L^{\infty}$. Together with the $C^{1}$ regularity of $v_{0}, \varphi_{0}$ by Lemmas 3 and 5 of [5] $v, \varphi$ are then bootstrapped to $C^{\alpha}(\bar{\Omega})$ for some $\alpha \in(0,1)$. Since $u=F^{-1}(v)$ and $F \in C^{2}$ under our assumptions about $\sigma$ the same is true of $u$. $\mathrm{Q}$

The result of [13] also guarantees uniqueness of solution when the boundary data $v_{0}, \varphi_{0}$ are sufficiently "small". Unfortunately, this does not extend here. Of course, there is uniqueness for each $\sigma_{m}$, but it is conceivable that (1.1) also has weak solutions that are not bounded away from $u_{*}$ even for small boundary data. They would not solve (1.1) with $\sigma_{m}$ in place of $\sigma$ for any $m<u_{*}$, and the Diesselhorst-Chen substitution would not be defined for them, so there would not be a corresponding $v$.

2.2. $L^{\infty}$ estimates. In this section we give a proof of Theorem 2.9. and therefore complete the proof of Theorem 2.1. This is accomplished through a series of lemmas following the general outline of [5]. By assumption on $\sigma$, we have $v=F(u)=$ $\int_{0}^{u} \frac{d s}{\sigma(s)} \rightarrow \infty$ as $u \rightarrow u_{*}$. Therefore, if we can show that $\|v\|_{\infty}<\infty$ not only will we have $\|u\|_{\infty}<\infty$ but also $0 \leq u \leq F^{-1}\left(\|v\|_{\infty}\right)<u_{*}$ a.e., that is $u$ is bounded away from the critical temperature everywhere in the domain.

Lemma 2.3. The function $a(v)$ is strictly positive, monotone decreasing, and

$$
e^{-\mu|y|} \leq \frac{a(v+y)}{a(v)} \leq e^{\mu|y|} .
$$

Moreover, for any $p \geq 2$, we have

$$
\frac{a(v)}{v^{p}} \int_{0}^{v} \frac{s^{p-2}}{a(s)} d s \underset{v \rightarrow \infty}{\longrightarrow} 0 .
$$

Proof. Since $F^{-1}([0, \infty))=\left[0, u_{*}\right)$ and $\sigma>0$ on $\left[0, u_{*}\right)$, it follows that $a(v)>0$. Since $\sigma \geq 0$ the function $F$ is monotone increasing, and therefore so is $F^{-1}$. As $\sigma$ is monotone decreasing and $a=\sigma \circ F^{-1}$, we have the same for $a$. To prove (2.5), consider

$$
\ln \frac{a(v+y)}{a(v)}=\ln a(v+y)-\ln a(v)=\int_{v}^{v+y}(\ln a(s))^{\prime} d s=\int_{v}^{v+y} \frac{a^{\prime}(s)}{a(s)} d s,
$$

where we assumed $y \geq 0$ for definiteness. Now by the chain rule

$$
\begin{aligned}
a^{\prime}(s)=a^{\prime}\left(F^{-1}(s)\right)\left(F^{-1}(s)\right)^{\prime}=\sigma^{\prime}\left(F^{-1}(s)\right) \frac{1}{F^{\prime}\left(F^{-1}(s)\right)} \\
\quad=\sigma^{\prime}\left(F^{-1}(s)\right) \frac{1}{1 / \sigma\left(F^{-1}(s)\right)}=\sigma^{\prime}\left(F^{-1}(s)\right) a(s) .
\end{aligned}
$$

Since $\left|\sigma^{\prime}\right| \leq \mu$ this implies $\left|\frac{a^{\prime}(s)}{a(s)}\right| \leq \mu$, and therefore

$$
\begin{aligned}
\left|\ln \frac{a(v+y)}{a(v)}\right| & \leq \int_{v}^{v+y} \mu d s=\mu|y|, \quad \text { or equivalently } \\
-\mu|y| & \leq \ln \frac{a(v+y)}{a(v)} \leq \mu|y| .
\end{aligned}
$$


Exponentiating the last inequality gives (2.5). The claim about the limit follows from the monotone decrease of $a(v)$. Namely, we have

$$
\frac{a(v)}{v^{p}} \int_{0}^{v} \frac{s^{p-2}}{a(s)} d s=\frac{1}{v^{p}} \int_{0}^{v} s^{p-2} \frac{a(v)}{a(s)} d s \leq \frac{1}{v^{p}} \int_{0}^{v} v^{p-2} \cdot 1 d s=\frac{1}{v} \underset{v \rightarrow \infty}{\longrightarrow} 0 .
$$

Due to non-vanishing of $a(v)$ we immediately have from the maximum principle that for any weak solution to (2.3), $v \geq 0$ and $\|\varphi\|_{\infty} \leq\left\|\varphi_{0}\right\|_{\infty}$. Note that we could not infer this from (1.1) directly since $\sigma(u)$ may be vanishing. The hard part is to obtain an $L^{\infty}$ estimate for $v$, and therefore, for $u=F^{-1}(v)$. To this end, we set $\psi:=\left(\varphi-\varphi_{0}\right)^{2}+v$. Then $\nabla \psi=2\left(\varphi-\varphi_{0}\right) \nabla\left(\varphi-\varphi_{0}\right)+\nabla v$, and $\frac{\partial \psi}{\partial n}=\frac{\partial v}{\partial n}, \psi=v$ on $\partial \Omega$ since $\varphi=\varphi_{0}$ on $\partial \Omega$. Although we write differential identities for simplicity here and below they should be understood in the weak form as in (2.1).

Lemma 2.4. For a weak solution $(v, \varphi)$ to (2.3) and $\psi:=\left(\varphi-\varphi_{0}\right)^{2}+v$, one has

$$
\nabla \cdot(a(v) \nabla \psi)=a(v)|\nabla \varphi|^{2}-2 \nabla \cdot\left(\left(\varphi-\varphi_{0}\right) a(v) \nabla \varphi_{0}\right)-2 a(v) \nabla \varphi \cdot \nabla \varphi_{0} .
$$

Moreover,

$$
-\nabla \cdot(a(v) \nabla \psi) \leq a(v)\left|\nabla \varphi_{0}\right|^{2}+2 \nabla \cdot\left(\left(\varphi-\varphi_{0}\right) a(v) \nabla \varphi_{0}\right)
$$

Proof. We write $a$ instead of $a(v)$ for short. Since $\nabla \psi=2\left(\varphi-\varphi_{0}\right) \nabla\left(\varphi-\varphi_{0}\right)+\nabla v$, by direct computation we obtain:

$$
\nabla \cdot(a \nabla \psi)=\nabla \cdot(a \nabla v)+2 \nabla \cdot\left(a\left(\varphi-\varphi_{0}\right) \nabla \varphi\right)-2 \nabla \cdot\left(a\left(\varphi-\varphi_{0}\right) \nabla \varphi_{0}\right) .
$$

Simplifying the middle term, we have

$2 \nabla \cdot\left(a\left(\varphi-\varphi_{0}\right) \nabla \varphi\right)=2\left(\varphi-\varphi_{0}\right) \nabla \cdot(a \nabla \varphi)+2 a \nabla \varphi \cdot \nabla\left(\varphi-\varphi_{0}\right)=0+2 a|\nabla \varphi|^{2}-2 a \nabla \varphi \cdot \nabla \varphi_{0}$

by the first equation in (2.3). By the second equation in (2.3): $\nabla \cdot(a \nabla v)=-a|\nabla \varphi|^{2}$, so that

$$
\nabla \cdot(a \nabla \psi)=-a|\nabla \varphi|^{2}+2 a|\nabla \varphi|^{2}-2 a \nabla \varphi \cdot \nabla \varphi_{0}-2 \nabla \cdot\left(\left(\varphi-\varphi_{0}\right) a \nabla \varphi_{0}\right),
$$

which yields the desired equation. To obtain the inequality, note that $-a|\nabla \varphi|^{2}+$ $2 a \nabla \varphi \cdot \nabla \varphi_{0} \leq a\left|\nabla \varphi_{0}\right|^{2}$ by applying $2 x y \leq x^{2}+y^{2}$ with $x=a^{1 / 2} \nabla \varphi$ and $y=a^{1 / 2} \nabla \varphi_{0}$. 口

Since $\psi=\left(\varphi-\varphi_{0}\right)^{2}+v$ and we already know that $\|\varphi\|_{\infty} \leq\left\|\varphi_{0}\right\|_{\infty}$ it suffices to show that $\|\psi\|_{\infty}<\infty$. In view of Lemma 2.4 we will be working with the inequality

$$
\begin{aligned}
-\nabla \cdot(a(v) \nabla \psi) & \leq a(v)\left|\nabla \varphi_{0}\right|^{2}+2 \nabla \cdot\left(a(v)\left(\varphi-\varphi_{0}\right) \nabla \varphi_{0}\right), \\
\psi & =F\left(u_{0}\right) \quad \text { on } \quad \Gamma_{D}, \\
\frac{\partial \psi}{\partial n} & +\frac{\beta}{a(v)}\left(F^{-1}(v)-u_{1}\right)=0 \quad \text { on } \quad \Gamma_{R} .
\end{aligned}
$$

Following Chen's suggestion in [5] we set $\xi(\psi):=\int_{M}^{\psi} \frac{s^{p-2}}{a(s)} d s$ (where $M$ is a positive constant to be specified later), multiply both sides of (2.6) by $\xi$, and integrate 
by parts. We set $\psi_{M}:=\max \{M, \psi\}$.

Lemma 2.5. For any $p \geq 2$ and $M>F\left(\left\|u_{0}\right\|_{\infty}\right)$, the following estimate holds

$$
\begin{aligned}
& \int_{\Omega} \frac{a(v)}{a\left(\psi_{M}\right)} \psi_{M}^{p-2}\left|\nabla \psi_{M}\right|^{2}+\int_{\Gamma_{M}} \beta \xi\left(\psi_{M}\right)\left(F^{-1}(v)-u_{1}\right) \\
& \quad \leq \int_{\Omega} \frac{a(v)}{a\left(\psi_{M}\right)}\left|\nabla \varphi_{0}\right|^{2} a\left(\psi_{M}\right) \xi\left(\psi_{M}\right)-2 \int_{\Omega} \frac{a(v)}{a(\psi)}\left(\varphi-\varphi_{0}\right) \psi_{M}^{p-2} \nabla \varphi_{0} \nabla \psi_{M},
\end{aligned}
$$

where $\Gamma_{M}:=\partial \Omega \cap\{\psi>M\}$

Proof. By the product rule,

$$
\nabla \cdot(a(v) \nabla \psi) \xi\left(\psi_{M}\right)=\nabla \cdot\left(a(v) \xi\left(\psi_{M}\right) \nabla \psi\right)-a(v) \nabla \psi \cdot \nabla \xi\left(\psi_{M}\right),
$$

and by definition of $\xi$, we have $\nabla \xi\left(\psi_{M}\right)=\frac{\psi_{M}^{p-2}}{a\left(\psi_{M}\right)} \nabla \psi_{M}$. Therefore,

$$
-\int_{\Omega} \nabla \cdot(a(v) \nabla \psi) \xi\left(\psi_{M}\right)=\int_{\Omega} \frac{a(v)}{a\left(\psi_{M}\right)} \psi_{M}^{p-2} \nabla \psi \cdot \nabla \psi_{M}-\int_{\partial \Omega} a(v) \xi\left(\psi_{M}\right) \frac{\partial \psi}{\partial n} .
$$

We now stipulate that $M>F\left(\left\|u_{0}\right\|_{\infty}\right)$. Then $\left.\psi\right|_{\Gamma_{D}}<M$ and $\left.\psi_{M}\right|_{\Gamma_{D}}=M$, $\left.\xi\left(\psi_{M}\right)\right|_{\Gamma_{D}}=0$. Hence, the boundary integral reduces to $\Gamma_{R}$, where $\psi=\psi_{M}$ and $\frac{\partial \psi}{\partial n}=-\frac{\beta}{a(v)}\left(F^{-1}(v)-u_{1}\right)$. We can also replace $\psi$ by $\psi_{M}$ in the interior integrals because $\xi\left(\psi_{M}\right)=0$ on the set where $\psi<\psi_{M}$. This leads to

$$
-\int_{\Omega} \nabla \cdot\left(a(v) \nabla \psi_{M}\right) \xi\left(\psi_{M}\right)=\int_{\Omega} \frac{a(v)}{a\left(\psi_{M}\right)} \psi_{M}^{p-2}\left|\nabla \psi_{M}\right|^{2}+\int_{\Gamma_{M}} \beta \xi\left(\psi_{M}\right)\left(F^{-1}(v)-u_{1}\right),
$$

where $\Gamma_{M} \subset \Gamma_{R}$ is the part of the boundary where $\psi>M$. Similarly,

$\nabla\left(a(v)\left(\varphi-\varphi_{0}\right) \xi\left(\psi_{M}\right) \nabla \varphi_{0}\right)=\nabla\left(a(v)\left(\varphi-\varphi_{0}\right) \nabla \varphi_{0}\right) \xi\left(\psi_{M}\right)+a(v)\left(\varphi-\varphi_{0}\right) \frac{\nabla \varphi_{0} \psi_{M}^{p-2}}{a\left(\psi_{M}\right)} \nabla \psi_{M}$,

and

$$
\begin{aligned}
\int_{\Omega} \nabla\left(a(v)\left(\varphi-\varphi_{0}\right) \nabla \varphi_{0}\right) \xi\left(\psi_{M}\right) & =\int_{\partial \Omega} a(v)\left(\varphi-\varphi_{0}\right) \xi\left(\psi_{M}\right) \frac{\partial \varphi_{0}}{\partial n} \\
& -\int_{\Omega} \frac{a(v)}{a\left(\psi_{M}\right)}\left(\varphi-\varphi_{0}\right) \nabla \varphi_{0} \psi_{M}^{p-2} \nabla \psi_{M},
\end{aligned}
$$

where the boundary term vanishes because $\left.\varphi\right|_{\partial \Omega}=0$. Since $\xi\left(\psi_{M}\right) \geq 0$ the inequality (2.6) yields (2.7) . $\mathrm{C}$

We now convert (2.7) into an estimate that will be used for bootstrapping $\psi_{M}$ (and hence $\psi$ ) into $L^{\infty}$. This involves further increase for $M$.

Lemma 2.6. Let $p \geq 2$ and $M>4\left\|\varphi_{0}\right\|_{\infty}^{2}+F\left(\left\|u_{0}\right\|_{\infty}\right)$. Then the following estimate holds

$$
\int_{\Omega} \psi_{M}^{p-2}\left|\nabla \psi_{M}\right|^{2} \leq \frac{8 e^{8 \mu\left\|\varphi_{0}\right\|_{\infty}^{2}\left\|\varphi_{0}\right\|_{W^{1, \infty}}^{2}}}{1-2 \varepsilon e^{8 \mu\left\|\varphi_{0}\right\|_{\infty}^{2}\left\|\varphi_{0}\right\|_{W^{1, \infty}}^{2}}} \int_{\Omega}\left(\varepsilon \psi_{M}^{p}+C_{\varepsilon}+\varepsilon^{1-p}\right),
$$

where $\varepsilon$ and $C_{\varepsilon}$ are chosen so that $a(v) \int_{0}^{v} \frac{s^{p-2}}{a(s)} d s \leq \varepsilon v^{p}+C_{\varepsilon}$ for all $v>0$.

Proof. Since $\psi>M$ on $\Gamma_{M}$, we have $v=\psi-\left(\varphi-\varphi_{0}\right)^{2}>\psi-4\left\|\varphi_{0}\right\|_{\infty}^{2}>$ $M-4\left\|\varphi_{0}\right\|_{\infty}^{2}>F\left(\left\|u_{1}\right\|_{\infty}\right)$, so $F^{-1}(v)>u_{1}$ a.e. on $\Gamma_{M}$, meaning that the boundary 
integral in (2.7) is positive. Moreover, $|\psi-v| \leq 4\left\|\varphi_{0}\right\|_{\infty}^{2}$, so on the part of $\Omega$ where $\xi\left(\psi_{M}\right) \neq 0$, we have $e^{-4 \mu\left\|\varphi_{0}\right\|_{\infty}^{2}} \leq \frac{a(v)}{a\left(\psi_{M}\right)} \leq e^{4 \mu\left\|\varphi_{0}\right\|_{\infty}^{2}}$ by Lemma 2.3. In view of this, (2.7) implies

$$
\begin{aligned}
e^{-4 \mu\left\|\varphi_{0}\right\|_{\infty}^{2}} \int_{\Omega} \psi_{M}^{p-2}\left|\nabla \psi_{M}\right|^{2} & \leq e^{4 \mu\left\|\varphi_{0}\right\|_{\infty}^{2}}\left(\left\|\varphi_{0}\right\|_{\infty}^{2} \int a\left(\psi_{M}\right) \xi\left(\psi_{M}\right)\right. \\
& \left.+2\left(2\left\|\varphi_{0}\right\|_{\infty}^{2}\right)\left\|\nabla \varphi_{0}\right\|_{\infty} \int_{\Omega} \psi_{M}^{p-2}\left|\nabla \psi_{M}\right|\right),
\end{aligned}
$$

and

$$
\int_{\Omega} \psi_{M}^{p-2}\left|\nabla \psi_{M}\right|^{2} \leq 4 e^{8 \mu\left\|\varphi_{0}\right\|_{\infty}^{2}}\left\|\varphi_{0}\right\|_{W^{1, \infty}}^{2}\left(\int_{\Omega} a\left(\psi_{M}\right) \xi\left(\psi_{M}\right)+\int_{\Omega} \psi_{M}^{p-2}\left|\nabla \psi_{M}\right|\right) .
$$

By Lemma 2.3,

$$
\frac{a(v) \xi(v)}{v^{p}}=\frac{a(v)}{v^{p}} \int_{M}^{v} \frac{s^{p-2}}{a(s)} d s \leq \frac{a(v)}{v^{p}} \int_{0}^{v} \frac{s^{p-2}}{a(s)} d s \underset{v \rightarrow \infty}{\longrightarrow} 0 .
$$

Therefore, $a(v) \xi(v) \leq \varepsilon v^{p}+C_{\varepsilon}$ for arbitrarily small $\varepsilon$ and suitable $C_{\varepsilon}$. Hence, $\int_{\Omega} a\left(\psi_{M}\right) \xi\left(\psi_{M}\right) \leq \int_{\Omega}\left(\varepsilon \psi_{M}^{p}+C_{\varepsilon}\right)$.

By the Cauchy inequality with $\varepsilon, x y \leq \frac{1}{2 \varepsilon} x^{2}+\frac{\varepsilon}{2} y^{2}$, applied to $x=\psi_{M}^{(p-2) / 2}$ and $y=\psi_{M}^{(p-2) / 2}|\nabla \psi|$, we have

$$
\psi_{M}^{p-2}|\nabla \psi| \leq \frac{1}{2 \varepsilon} \psi_{M}^{p-2}+\frac{\varepsilon}{2} \psi_{M}^{p-2}\left|\nabla \psi_{M}\right|^{2} .
$$

Finally, applying the Young inequality, $x y \leq \frac{x^{\alpha}}{\alpha}+\frac{x^{\beta}}{\beta}$ for $\frac{1}{\alpha}+\frac{1}{\beta}=1, \alpha, \beta>0$, with $x=\frac{1}{2 \varepsilon \delta}, y=\delta\left|\psi_{M}^{p-2}\right|$, we obtain $\frac{1}{2 \varepsilon} \psi_{M}^{p-2} \leq \frac{1}{\alpha} \frac{1}{(2 \varepsilon \delta)^{\alpha}}+\frac{1}{\beta}\left(\delta \psi_{M}^{p-2}\right)^{\beta}$. Now select $\alpha$ and $\beta$ so that $(p-2) \beta=p$, that is, $\beta:=p /(p-2)$ and $\alpha:=p / 2$. Then we have

$$
\frac{1}{2 \varepsilon} \psi_{M}^{p-2} \leq \frac{2}{p} \frac{1}{(2 \varepsilon \delta)^{p / 2}}+\left(1-\frac{2}{p}\right) \delta^{\frac{p}{p-2}} \psi_{M}^{p} \leq \frac{1}{(\varepsilon \delta)^{(p / 2)}}+\delta^{p /(p-2)} \psi_{M}^{p}
$$

since $p \geq 2$. We now set $\delta^{p /(p-2)}=\varepsilon$ so that $(\varepsilon \delta)^{p / 2}=\varepsilon^{p / 2}\left(\varepsilon^{(p-2) / p}\right)^{p / 2}=\varepsilon^{p-1}$, producing $\frac{1}{2 \varepsilon} \psi_{M}^{p-2} \leq \varepsilon \psi_{M}^{p}+\varepsilon^{1-p}$. Thus,

$$
\begin{aligned}
& \int_{\Omega} \psi_{M}^{p-2}\left|\nabla \psi_{M}\right|^{2} \leq 4 e^{8 \mu\left\|\varphi_{0}\right\|_{\infty}^{2}}\left\|\varphi_{0}\right\|_{W^{1, \infty}}^{2}\left(\int_{\Omega} 2 \varepsilon \psi_{M}^{p}\right. \\
& \left.+\int_{\Omega}\left\{\frac{\varepsilon}{2} \psi_{M}^{p-2}\left|\nabla \psi_{M}\right|^{2}+C_{\varepsilon}+\varepsilon^{1-p}\right\}\right),
\end{aligned}
$$

which is a rearragement of (2.8).

$$
\text { Set } C:=\frac{8 e^{8 \mu\left\|\varphi_{0}\right\|_{\infty}^{2}}\left\|\varphi_{0}\right\|_{W^{1, \infty}}^{2}}{1-2 \varepsilon e^{8 \mu\left\|\varphi_{0}\right\|_{\infty}^{2}\left\|\varphi_{0}\right\|_{W^{1, \infty}}^{2}}}
$$

COROLlary 2.7. The following estimates hold

$$
\begin{aligned}
\left\|\psi_{M}\right\|_{2}^{2} & \leq \frac{2 \operatorname{mes}(\Omega)}{1-2 \varepsilon C C_{D}^{2}}\left\{C C_{D}^{2}\left(C_{\varepsilon}+\varepsilon^{-1}\right)+M^{2} \operatorname{mes}(\Omega)\right\}\left\|\nabla \psi_{M}\right\|_{2}^{2} \\
& \leq \varepsilon C\left\|\psi_{M}\right\|_{2}^{2}+C\left(C_{\varepsilon}+\varepsilon^{-1}\right) \operatorname{mes}(\Omega)
\end{aligned}
$$


where $C_{D}:=C\left(\Omega, \Gamma_{D}\right)$ is a constant from the Poincaré inequality.

Proof. With $p=2$ in (2.8), we have $\int_{\Omega}\left|\nabla \psi_{M}\right|^{2} \leq C \int_{\Omega}\left(\varepsilon \psi_{M}^{2}+C_{\varepsilon}+\varepsilon^{-1}\right)$. Since $\psi=F\left(u_{0}\right)$ and $M>F\left(\left\|u_{0}\right\|_{\infty}\right)$, we have that $\psi_{M}=\max \{\psi, M\}=M$ on $\Gamma_{D}$, and therefore, $\psi_{M}-M=0$ on $\Gamma_{D}$. By the Poincaré inequality, there exists $C_{D}:=$ $C\left(\Omega, \Gamma_{D}\right)$ such that

$$
\left\|\psi_{M}-M\right\|_{2} \leq C_{D}\left\|\nabla\left(\psi_{M}-M\right)\right\|_{2}=C_{D}\left\|\nabla \psi_{M}\right\|_{2},
$$

and therefore, $\left\|\psi_{M}\right\|_{2} \leq C_{D}\left\|\nabla \psi_{M}\right\|_{2}+M \operatorname{mes}(\Omega)$. Thus, we obtain

$$
\left\|\psi_{M}\right\|_{2}^{2} \leq 2 C_{D}^{2}\left\|\nabla \psi_{M}\right\|_{2}^{2}+2 M^{2} \operatorname{mes}^{2}(\Omega) \leq 2 C C_{D}^{2} \int_{\Omega}\left(\varepsilon \psi_{M}^{2}+C_{\varepsilon}+\varepsilon^{-1}\right)+2 M^{2} \operatorname{mes}^{2}(\Omega) .
$$

This implies

$$
\left\|\psi_{M}\right\|_{2}^{2} \leq \frac{2 \operatorname{mes}(\Omega)}{1-2 \varepsilon C C_{D}^{2}}\left\{C C_{D}^{2}\left(C_{\varepsilon}+\varepsilon^{-1}\right)+M^{2} \operatorname{mes}(\Omega)\right\}\left\|\nabla \psi_{M}\right\|_{2}^{2},
$$

and the second estimate is obvious from (2.8) with $p=2$. $\mathrm{u}$

Note that in Lemma 2.6 the choice of $\varepsilon$ does not depend on $M$, and the estimates (2.8), (2.9) are valid for $\psi_{M}$ with any $M>4\left\|\varphi_{0}\right\|_{\infty}^{2}+F\left(\left\|u_{0,1}\right\|_{\infty}\right)$.

Corollary 2.8. Let $M>\max \left\{1, C_{\varepsilon}, 1 / \varepsilon, 4\left\|\varphi_{0}\right\|_{\infty}^{2}+F\left(\left\|u_{0,1}\right\|_{\infty}\right)\right\}$. Then, for any $p \geq 2$, we have

$$
\int_{\Omega} \psi_{M}^{p-2}\left|\nabla \psi_{M}\right|^{2} \leq C(M+\varepsilon) \int_{\Omega} \psi_{M}^{p}
$$

Proof. With our choice of $M, C_{\varepsilon}+\varepsilon^{1-p}=C_{\varepsilon}+\varepsilon \frac{1}{\varepsilon^{p}} \leq 2 M^{p} \leq 2 \psi_{M}^{p}$. Substituting this in (2.9) yields the claim. $\square$

Now, we are ready for the main theorem of this section.

TheOrem 2.9. Let $(v, \varphi)$ be a solution to (2.3). Then

$$
\begin{aligned}
\|v\|_{\infty} & \leq 4\left\|\varphi_{0}\right\|_{\infty}^{2}+\max \left\{M,\left\|\psi_{M}\right\|_{\infty}\right\} \\
\left\|\psi_{M}\right\|_{\infty} & \leq\left\|\psi_{M}\right\|_{2} \cdot\left\{\begin{array}{l}
2 C_{2}, d \leq 2, \\
\left(2 C_{2}\right)^{d / 2}\left(\frac{d}{d-2}\right)^{d(d-2) / 4}, d>2
\end{array}\right.
\end{aligned}
$$

where $C_{2}:=\frac{1}{2} C_{1}\left(1+C^{1 / 2}(M+\varepsilon)^{1 / 2}\right), C_{1}$ is the constant from a Sobolev embedding theorem for $\left(\Omega, \Gamma_{D}\right)$, and where $\varepsilon, C$, and $M$ are defined in Lemma [2.6. (2.10), and Corollary 2.8, respectively, and $\left\|\psi_{M}\right\|_{2}$ is estimated in Corollary 2.7. In particular, $v, \psi$ satisfy a priori $L^{\infty}$ estimates.

Proof. Since $v=-\left(\varphi-\varphi_{0}\right)^{2}+\psi$ and $\psi_{M}=\max \{\psi, M\}$ we clearly have $\|v\|_{\infty} \leq$ $\max \left\{M,\left\|\psi_{M}\right\|_{\infty}\right\}+4\left\|\varphi_{0}\right\|_{\infty}^{2}$, and it remains to estimate $\left\|\psi_{M}\right\|_{\infty}$. It follows from a Sobolev embedding theorem for $q \leq 2 d /(d-2)$ when $d>2$, and any $1 \leq q<\infty$ when $d \leq 2$, that $\|\phi\|_{q} \leq C_{1}\|\phi\|_{H^{1}}=C_{1}\left(\|\phi\|_{2}+\|\nabla \phi\|_{2}\right)$. Let us apply this to $\phi=$ $\psi_{M}^{p / 2}$, noting that $\nabla \phi=(p / 2) \psi_{M}^{(p / 2)-1} \nabla \psi_{M}$, and $\|\nabla \phi\|_{2}^{2}=\left(p^{2} / 4\right) \int_{\Omega} \psi_{M}^{p-2}\left|\nabla \psi_{M}\right|^{2} \leq$ $\left(p^{2} / 4\right) C(M+\varepsilon)\left\|\psi_{M}\right\|_{p}^{p}$ by Corollary 2.8. Similarly, $\|\phi\|_{2}^{2}=\left\|\psi_{M}\right\|_{p}^{p}$, and $\|\phi\|_{q}=$ $\left(\int_{\Omega}\left|\psi_{M}^{p / 2}\right|^{q}\right)^{1 / q}=\left(\int_{\Omega} \psi_{M}^{m p}\right)^{1 / 2 m}=\left\|\psi_{M}\right\|_{m p}^{p / 2}$, where $m:=d /(d-2)=q / 2$ for $d>2$ and any $q \in(1, \infty)$ for $d \leq 2$. We have

$$
\begin{aligned}
\left\|\psi_{M}\right\|_{m p}^{p / 2} & \leq C_{1}\left(\left\|\psi_{M}\right\|_{p}^{p / 2}+(p / 2) C^{1 / 2}(M+\varepsilon)^{1 / 2}\left\|\psi_{M}\right\|_{p}^{p / 2}\right) \\
& \leq C_{1}\left(1+(p / 2) C^{1 / 2}(M+\varepsilon)^{1 / 2}\right)\left\|\psi_{M}\right\|_{p}^{p / 2}
\end{aligned}
$$


and since $p \geq 2$

$$
\left\|\psi_{M}\right\|_{m p}^{p / 2} \leq\left(\frac{1}{2} C_{1}\left(1+C^{1 / 2}(M+\varepsilon)^{1 / 2}\right) p\right)^{2 / p}\left\|\psi_{M}\right\|_{p}=\left(C_{2} p\right)^{2 / p}\left\|\psi_{M}\right\|_{p}
$$

where we introduced $C_{2}:=\frac{1}{2} C_{1}\left(1+C^{1 / 2}(M+\varepsilon)^{1 / 2}\right)$. Since $m>1$, this sets up a bootstrap starting with $p=2$ and proceeding through $2 m, 2 m^{2}, 2 m^{3}, \ldots, 2 m^{k} \rightarrow \infty$ as $k \rightarrow \infty$. In particular,

$$
\begin{aligned}
& \left\|\psi_{M}\right\|_{2 m^{k}} \leq\left(2 C_{2} m^{k-1}\right)^{2 / 2 m^{k-1}} \ldots\left(2 C_{2}\right)^{2 / 2}\left\|\psi_{M}\right\|_{2}
\end{aligned}
$$

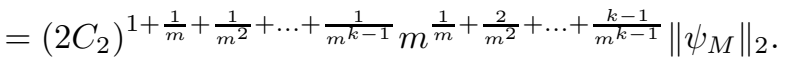

But $1+\frac{1}{m}+\frac{1}{m^{2}}+\ldots+\frac{1}{m^{k-1}} \leq \frac{1}{1-\frac{1}{m}}$ and $\frac{1}{m}+\frac{2}{m^{2}}+\ldots+\frac{k-1}{m^{k-1}}=\frac{1}{m} \frac{1}{\left(1-\frac{1}{m}\right)^{2}}$, so that

$$
\left\|\psi_{M}\right\|_{2 m^{k}} \leq\left(2 C_{2}\right)^{m /(m-1)} m^{m /(m-1)^{2}}\left\|\psi_{M}\right\|_{2}
$$

Now letting $k \rightarrow \infty$ in (2.13) we obtain $\left\|\psi_{M}\right\|_{\infty}$ on the left, then setting $m=d /(d-2)$ for $d>2$, and $m \rightarrow \infty$ for $d \leq 2$, will produce

$$
\left\|\psi_{M}\right\|_{\infty} \leq\left\{\begin{array}{l}
2 C_{2}\left\|\psi_{M}\right\|_{2}, d \leq 2 \\
\left(2 C_{2}\right)^{d / 2}\left(\frac{d}{d-2}\right)^{d(d-2) / 4}\left\|\psi_{M}\right\|_{2}, d>2
\end{array}\right.
$$

as desired. This concludes our proof of the $L^{\infty}$ estimate for $\psi$ since $\psi=\left(\varphi-\varphi_{0}\right)^{2}+$ $F(u)$, and we already have one for $\varphi$.

3. Further a priori estimates. In order to be able to prove existence of optimal control we need to derive more a priori estimates. In what follows, given $\beta \in U_{\mathcal{M}}$, we denote the solution to (2.1) by $u(\beta)$ and $\varphi(\beta)$.

Theorem 3.1. Let $\beta \in U_{\mathcal{M}}$ be given. Then $u(\beta)$ and $\varphi(\beta)$ solving (2.1) satisfy

$$
\begin{aligned}
\|\varphi\|_{W^{1, r}(\Omega)} & \leq \Phi \text { for some } r>2, \text { and } \\
\|u\|_{H^{1}(\Omega)} & \leq \tilde{C}
\end{aligned}
$$

where $\Phi$ and $\tilde{C}$ are some positive constants.

Proof. First, we show the estimate for $\varphi$. We are given the solution of a nonhomogeneous Dirichlet problem

$$
\begin{aligned}
\nabla \cdot(\sigma(u) \nabla \varphi) & =0 \text { in } \Omega, \\
\varphi & =\varphi_{0} \text { on } \partial \Omega .
\end{aligned}
$$

Because of the assumption 2 from the Introduction we treat $\sigma(u)$ in (1.1) as a bounded coefficient. Consider the following Dirichlet problem with zero boundary data

$$
\begin{aligned}
-\nabla \cdot(\sigma(u) \nabla \tilde{\varphi}) & =\nabla \cdot\left(\sigma(u) \nabla \varphi_{0}\right) \text { in } \Omega, \\
\tilde{\varphi} & =0 \text { on } \partial \Omega .
\end{aligned}
$$

By the standard theory for elliptic equations in divergence form, it follows that there exists $\tilde{\varphi}=\left(\varphi-\varphi_{0}\right) \in H_{0}^{1}(\Omega)$ that solves (3.3). By Theorem 2.2. $\tilde{\varphi} \in W_{0}^{1, r}(\Omega)$ and

$$
\|\tilde{\varphi}\|_{W_{0}^{1, r}(\Omega)} \leq C\left\|\sigma(u) \nabla \varphi_{0}\right\|_{r} \leq C \mu\left\|\nabla \varphi_{0}\right\|_{r} \quad \text { for each } 2<r<\infty
$$


Since $\|\varphi\|_{W^{1, r}(\Omega)} \leq\left\|\varphi-\varphi_{0}\right\|_{W^{1, r}(\Omega)}+\left\|\varphi_{0}\right\|_{W^{1, r}(\Omega)}$ and $\left\|\varphi-\varphi_{0}\right\|_{W^{1, r}(\Omega)} \equiv\|\tilde{\varphi}\|_{W^{1, r}(\Omega)} \leq$ $C^{\prime}\|\tilde{\varphi}\|_{W_{0}^{1, r}(\Omega)} \leq C^{\prime} C \mu\left\|\nabla \varphi_{0}\right\|_{r}$, and taking into account that $\left\|\nabla \varphi_{0}\right\|_{r} \leq C_{3}\left\|\nabla \varphi_{0}\right\|_{\infty}$ as well as $\left\|\varphi_{0}\right\|_{W^{1, r}(\Omega)} \leq C_{4}\left\|\varphi_{0}\right\|_{W^{1, \infty}(\Omega)}$, it is easy to see that

$$
\|\varphi\|_{W^{1, r}(\Omega)} \leq \Phi \text { for each } 2<r<\infty
$$

where $\Phi \stackrel{\text { def }}{=} C^{\prime} C C_{3} \mu\left\|\nabla \varphi_{0}\right\|_{\infty}+C_{4}\left\|\varphi_{0}\right\|_{W^{1, \infty}(\Omega)}$. Now we derive the estimate for $\|u\|_{H^{1}(\Omega)}$. From the weak formulation (2.1), we can write

$$
\begin{array}{r}
\int_{\Omega}\left(\nabla\left(u-u_{0}\right)+\nabla u_{0}\right) \nabla v d x+\int_{\Gamma_{R}} \beta\left(u-u_{0}\right) v d s+\int_{\Gamma_{R}} \beta\left(u_{0}-u_{1}\right) v d s \\
=\int_{\Omega}\left(\varphi_{0}-\varphi\right) \sigma(u) \nabla \varphi \nabla v d x+\int_{\Omega} \sigma(u) v \nabla \varphi \nabla \varphi_{0} d x
\end{array}
$$

Substituting $v=u-u_{0}$ as a test function into (3.4), we obtain

$$
\begin{aligned}
& \int_{\Omega}\left|\nabla\left(u-u_{0}\right)\right|^{2}+\int_{\Gamma_{R}} \beta\left(u-u_{0}\right)^{2}=\int_{\Omega} \nabla\left(u_{0}-u\right) \nabla u_{0} \\
+ & \int_{\Gamma_{R}} \beta\left(u_{1}-u_{0}\right)\left(u-u_{0}\right)+\int_{\Omega}\left(\varphi_{0}-\varphi\right) \sigma(u) \nabla \varphi \nabla\left(u-u_{0}\right)+\int_{\Omega} \sigma(u)\left(u-u_{0}\right) \nabla \varphi \nabla \varphi_{0} .
\end{aligned}
$$

Since $\beta(x) \geq 0$, the left hand side of (3.5) can be written as

$$
\int_{\Omega}\left|\nabla\left(u-u_{0}\right)\right|^{2} \leq \int_{\Omega}\left|\nabla\left(u-u_{0}\right)\right|^{2}+\int_{\Gamma_{R}} \beta\left(u-u_{0}\right)^{2} .
$$

Taking into account that $\beta \leq \mathcal{M}$ a.e., $\sup _{\Omega} \varphi \leq \tilde{M}$, and the trace inequality $\|u\|_{L^{2}\left(\Gamma_{R}\right)} \leq$ $M_{2}\|u\|_{H^{1}(\Omega)}$ (since $u-u_{0}=0$ on $\Gamma_{D}$ ), the right hand side of (3.5) can be estimated as follows

$$
\begin{aligned}
& \int_{\Omega} \nabla\left(u_{0}-u\right) \nabla u_{0}+\int_{\Gamma_{R}} \beta\left(u_{1}-u_{0}\right)\left(u-u_{0}\right)+\int_{\Omega}\left(\varphi_{0}-\varphi\right) \sigma(u) \nabla \varphi \nabla\left(u-u_{0}\right) \\
+ & \int_{\Omega} \sigma(u)\left(u-u_{0}\right) \nabla \varphi \nabla \varphi_{0} \leq\left\|u_{0}-u\right\|_{2}\left\|\nabla u_{0}\right\|_{2}+\mathcal{M} \sup _{\Gamma_{R}}\left|u_{1}-u_{0}\right| m e s^{1 / 2}\left(\Gamma_{R}\right) M_{2}\left\|u_{0}-u\right\|_{H^{1}} \\
+ & 2 \mu \tilde{M}\|\nabla \varphi\|_{2}\left\|\nabla\left(u_{0}-u\right)\right\|_{2}+\mu\left\|\nabla \varphi_{0}\right\|_{\infty}\left\|u_{0}-u\right\|_{2}\|\nabla \varphi\|_{2} \leq \tilde{C}_{1}\left\|u_{0}-u\right\|_{H^{1}(\Omega)} .
\end{aligned}
$$

Hence, we have

$$
\int_{\Omega}\left|\nabla\left(u-u_{0}\right)\right|^{2} d x \leq \tilde{C}_{1}\left\|u-u_{0}\right\|_{H^{1}(\Omega)} .
$$

Now, using an extension of the Poincaré inequality (as given by Theorem 5.8 in [20]), we obtain

$$
k\left\|u-u_{0}\right\|_{H^{1}(\Omega)}^{2} \leq\left\|\nabla\left(u-u_{0}\right)\right\|_{2}^{2} \leq \tilde{C}_{1}\left\|u-u_{0}\right\|_{H^{1}(\Omega)},
$$

which gives the desired estimate for $\|u\|_{H^{1}(\Omega)}$. $\square$ 
4. Existence of an optimal control. After we obtained $r$ and $s$ from (2.2), and the corresponding a priori estimates, we are in a position to prove existence of an optimal control.

THEOREM 4.1. There exists a solution to the optimal control problem (1.2).

Proof. We follow [14] closely. Choose a minimizing sequence $\left\{\beta_{n}\right\}_{n=1}^{\infty} \subset U_{\mathcal{M}}$ such that

$$
\lim _{n \rightarrow \infty} J\left(\beta_{n}\right)=\inf _{\beta \in U_{\mathcal{M}}} J(\beta) .
$$

Let $u_{n}=u\left(\beta_{n}\right)$ and $\varphi_{n}=\varphi\left(\beta_{n}\right)$ be the corresponding solutions to

$$
\begin{aligned}
\int_{\Omega} \nabla u_{n} \nabla v d x+\int_{\Gamma_{R}} \beta_{n}\left(u_{n}-u_{1}\right) v d s & =\int_{\Omega}\left(\varphi_{0}-\varphi_{n}\right) \sigma\left(u_{n}\right) \nabla \varphi_{n} \nabla v d x \\
& +\int_{\Omega} \sigma\left(u_{n}\right) \nabla \varphi_{n} \nabla \varphi_{0} v d x \quad \forall v \in V_{D}(\Omega) \\
\int_{\Omega} \sigma\left(u_{n}\right) \nabla \varphi_{n} \cdot \nabla w d x & =0 \quad \varphi_{n}-\varphi_{0} \in H_{0}^{1}(\Omega), u_{n}-u_{0} \in V_{D}, \forall w \in H_{0}^{1}(\Omega) .
\end{aligned}
$$

By Theorem 3.1 we have $\left\|u_{n}\right\|_{H^{1}(\Omega)} \leq C,\left\|\varphi_{n}\right\|_{W^{1, r}(\Omega)} \leq C$ for all $n$, where $C>0$ denotes a generic constant independent of $n$. Therefore, on a subsequence

$$
u_{n} \stackrel{w}{\longrightarrow} u^{*} \text { in } H^{1}(\Omega) \text { and } \varphi_{n} \stackrel{w}{\longrightarrow} \varphi^{*} \text { in } W^{1, r}(\Omega) .
$$

Also, $\beta_{n} \in U_{\mathcal{M}}$ for all $n$ implies that $\beta_{n} \in L^{\infty}\left(\Gamma_{R}\right)$ and $\left\|\beta_{n}\right\|_{L^{\infty}\left(\Gamma_{R}\right)} \leq \mathcal{M}$ for all $n$. Hence, on a subsequence $\beta_{n} \stackrel{w *}{\longrightarrow} \beta^{*}$ in $L^{\infty}\left(\Gamma_{R}\right)$. On the other hand, $\beta_{n} \in L^{2}\left(\Gamma_{R}\right)$ and $\left\|\beta_{n}\right\|_{L^{2}\left(\Gamma_{R}\right)} \leq \mathcal{M}$ for each $n$ implies $\beta_{n} \stackrel{w}{\rightarrow} \beta^{*}$ in $L^{2}\left(\Gamma_{R}\right)$.

As mentioned in Section 2, we have that $H^{1}(\Omega) \subset \subset L^{s}(\Omega)$, and since $r>d$, $W^{1, r}(\Omega) \subset \subset C(\bar{\Omega})$, where $r$ and $s$ were chosen in (2.2). Hence, on a subsequence

$$
\begin{aligned}
& \varphi_{n} \stackrel{s}{\rightarrow} \varphi^{*} \text { in } C(\bar{\Omega}), \nabla \varphi_{n} \stackrel{w}{\rightarrow} \nabla \varphi^{*} \text { in } L^{r}(\Omega), \\
& u_{n} \stackrel{s}{\rightarrow} u^{*} \text { in } L^{s}(\Omega), \nabla u_{n} \stackrel{w}{\longrightarrow} \nabla u^{*} \text { in } L^{2}(\Omega), \\
& \beta_{n} \stackrel{w}{\longrightarrow} \beta^{*} \text { in } L^{2}\left(\Gamma_{R}\right), \beta_{n} \stackrel{w *}{\longrightarrow} \beta^{*} \text { in } L^{\infty}\left(\Gamma_{R}\right) .
\end{aligned}
$$

Now we need to show that $u^{*}=u\left(\beta^{*}\right)$ and $\varphi^{*}=\varphi\left(\beta^{*}\right)$ solve (2.1) with control $\beta^{*}$, i.e., pass to the limit as $n \rightarrow \infty$ in (4.1). From (4.2) it is immediate that

$$
\int_{\Omega} \nabla u_{n} \cdot \nabla v d x \rightarrow \int_{\Omega} \nabla u^{*} \cdot \nabla v d x \text { as } n \rightarrow \infty .
$$

Using the trace inequality, the fact that $H^{1}(\Omega) \subset \subset L^{2}(\partial \Omega)$ and $\beta_{n} \stackrel{w *}{\longrightarrow} \beta^{*}$ in $L^{\infty}\left(\Gamma_{R}\right)$ we can show that

$$
\int_{\Gamma_{R}} \beta_{n} u_{n} v d s \rightarrow \int_{\Gamma_{R}} \beta^{*} u^{*} v d s \text { as } n \rightarrow \infty
$$

Next we show that

$$
\int_{\Omega} \sigma\left(u_{n}\right) v \nabla \varphi_{n} \cdot \nabla \varphi_{0} d x \rightarrow \int_{\Omega} \sigma\left(u^{*}\right) v \nabla \varphi^{*} \cdot \nabla \varphi_{0} d x \text { as } n \rightarrow \infty .
$$


We have

$$
\begin{aligned}
& \left|\int_{\Omega} \sigma\left(u_{n}\right) v \nabla \varphi_{n} \cdot \nabla \varphi_{0} d x-\int_{\Omega} \sigma\left(u^{*}\right) v \nabla \varphi^{*} \cdot \nabla \varphi_{0} d x\right| \\
\leq & \left|\int_{\Omega}\left[\sigma\left(u_{n}\right)-\sigma\left(u^{*}\right)\right] v \nabla \varphi_{n} \cdot \nabla \varphi_{0} d x\right|+\left|\int_{\Omega} \sigma\left(u^{*}\right) v\left(\nabla \varphi_{n}-\nabla \varphi^{*}\right) \cdot \nabla \varphi_{0} d x\right| \\
\leq & K\left\|\nabla \varphi_{0}\right\|_{\infty} \int_{\Omega}\left|u_{n}-u^{*}\right| \cdot|v| \cdot\left|\nabla \varphi_{n}\right| d x+\left|\int_{\Omega} \sigma\left(u^{*}\right) v\left(\nabla \varphi_{n}-\nabla \varphi^{*}\right) \cdot \nabla \varphi_{0} d x\right| \\
\leq & K\left\|\nabla \varphi_{0}\right\|_{\infty}\left\|u_{n}-u^{*}\right\|_{s} \cdot\|v\|_{2} \cdot\left\|\nabla \varphi_{n}\right\|_{r}+\left|\int_{\Omega} \sigma\left(u^{*}\right) v\left(\nabla \varphi_{n}-\nabla \varphi^{*}\right) \cdot \nabla \varphi_{0} d x\right| \rightarrow 0,
\end{aligned}
$$

where $K$ is the Lipschitz constant for $\sigma$, and where we took into account that $u_{n} \stackrel{s}{\rightarrow} u^{*}$ in $L^{s}(\Omega), \nabla \varphi \stackrel{w}{\rightarrow} \nabla \varphi^{*}$ in $L^{2}(\Omega)$, and $\left\|\nabla \varphi_{n}\right\|_{r} \leq C$. This completes the proof of (4.4). Similarly, using the corresponding convergences from (4.2) we can show that

$$
\begin{array}{r}
\int_{\Omega}\left(\varphi_{0}-\varphi_{n}\right) \sigma\left(u_{n}\right) \nabla \varphi_{n} \cdot \nabla v d x \rightarrow \int_{\Omega}\left(\varphi_{0}-\varphi^{*}\right) \sigma\left(u^{*}\right) \nabla \varphi^{*} \cdot \nabla v d x \\
\int_{\Omega} \sigma\left(u_{n}\right) \nabla \varphi_{n} \cdot \nabla w d x \rightarrow \int_{\Omega} \sigma\left(u^{*}\right) \nabla \varphi^{*} \cdot \nabla w d x \text { as } n \rightarrow \infty .
\end{array}
$$

More details on the above convergences can be found in [14]. Now letting $n \rightarrow \infty$ in (4.1), we obtain

$$
\begin{aligned}
\int_{\Omega} \nabla u^{*} \cdot \nabla v d x+\int_{\Gamma_{R}} \beta^{*} u^{*} v d x & =\int_{\Omega}\left(\varphi_{0}-\varphi^{*}\right) \sigma\left(u^{*}\right) \nabla \varphi^{*} \cdot \nabla v d x \\
& +\int_{\Omega} \sigma\left(u^{*}\right) \nabla \varphi^{*} \cdot \nabla \varphi_{0} v d x \forall v \in H^{1}(\Omega) \\
\int_{\Omega} \sigma\left(u^{*}\right) \nabla \varphi^{*} \cdot \nabla w d x & =0 \quad \varphi_{0}-\varphi_{n} \in H_{0}^{1}(\Omega), \forall w \in H_{0}^{1}(\Omega) .
\end{aligned}
$$

Therefore $\left(u^{*}, \varphi^{*}\right)$ is a weak solution associated with $\beta^{*}: u^{*}=u\left(\beta^{*}\right)$ and $\varphi^{*}=\varphi\left(\beta^{*}\right)$. Now we show that $\beta^{*}$ is optimal. As $\lim _{n \rightarrow \infty} J\left(\beta_{n}\right)$ exists we conclude $\lim _{n \rightarrow \infty} \int_{\Gamma_{R}} \beta_{n}^{2} d s$ exists and

$$
\begin{aligned}
\inf _{\beta \in U_{M}} J(\beta) & =\lim _{n \rightarrow \infty} J\left(\beta_{n}\right)=\lim _{n \rightarrow \infty} \int_{\Omega} u_{n} d x+\lim _{n \rightarrow \infty} \int_{\Gamma_{R}} \beta_{n}^{2} d s \\
& \geq \int_{\Omega} u^{*} d x+\int_{\Gamma_{R}}\left(\beta^{*}\right)^{2} d s=J\left(\beta^{*}\right) .
\end{aligned}
$$

This implies that $\beta^{*}$ is an optimal control.

5. Derivation of the optimality system. Our optimal control will be represented in terms of the solution to the optimality system, which consists of the original state system and the adjoint system whose construction, loosely speaking, follows the standard technique of (i) deriving the sensitivity equations (linearizing the state equations), (ii) exchanging the role of test function and solution in the linearization, and (iii) using the derivative of the cost function with respect to the state as a nonhomogeneity.

To obtain the necessary conditions for the optimality system we differentiate the objective functional with respect to the control. Since the objective functional 
depends on $u$, and $u$ is coupled to $\varphi$ through a PDE, we will need to differentiate $u$ and $\varphi$ with respect to the control $\beta$.

Theorem 5.1. (Sensitivities) If the boundary data $\varphi_{0}$ are sufficiently small, i.e., if $\left\|\varphi_{0}\right\|_{W^{1, \infty}(\Omega)}$ is small enough, then the mapping $\beta \mapsto(u, \varphi)$ is differentiable in the following sense:

$$
\begin{aligned}
& \frac{u(\beta+\varepsilon \ell)-u(\beta)}{\varepsilon} \stackrel{w}{\longrightarrow} \psi_{1} \text { in } H^{1}(\Omega), \\
& \frac{\varphi(\beta+\varepsilon \ell)-\varphi(\beta)}{\varepsilon} \stackrel{w}{\longrightarrow} \psi_{2} \text { in } H_{0}^{1}(\Omega) \text { as } \varepsilon \rightarrow 0
\end{aligned}
$$

for any $\beta \in U_{\mathcal{M}}$ and $\ell \in L^{\infty}(\partial \Omega)$ such that $(\beta+\varepsilon \ell) \in U_{\mathcal{M}}$ for small $\varepsilon$. Moreover, the sensitivities, $\psi_{1} \in H^{1}(\Omega)$ and $\psi_{2} \in H_{0}^{1}(\Omega)$, satisfy

$$
\begin{aligned}
\Delta \psi_{1}+\sigma^{\prime}(u)|\nabla \varphi|^{2} \psi_{1}+2 \sigma(u) \nabla \varphi \cdot \nabla \psi_{2} & =0 \text { in } \Omega, \\
\nabla \cdot\left[\sigma^{\prime}(u) \psi_{1} \nabla \varphi+\sigma(u) \nabla \psi_{2}\right] & =0 \text { in } \Omega, \\
\frac{\partial \psi_{1}}{\partial n}+\beta \psi_{1}+\ell\left(u-u_{1}\right) & =0 \text { on } \Gamma_{R}, \\
\psi_{1} & =0 \text { on } \Gamma_{D}, \\
\psi_{2} & =0 \text { on } \partial \Omega .
\end{aligned}
$$

Proof. We follow 14] with appropriate modifications where necessary. Earlier we denoted $u=u(\beta)$ and $\varphi=\varphi(\beta)$. Denote also $u^{\varepsilon}=u\left(\beta^{\varepsilon}\right), \varphi^{\varepsilon}=\varphi\left(\beta^{\varepsilon}\right)$, where $\beta^{\varepsilon} \stackrel{\text { def }}{=} \beta+\varepsilon \ell$. The weak formulation for $\left(u^{\varepsilon}, \varphi^{\varepsilon}\right)$ is

$$
\begin{aligned}
\int_{\Omega} \nabla u^{\varepsilon} \nabla v d x+\int_{\Gamma_{R}} \beta^{\varepsilon}\left(u^{\varepsilon}-u_{1}\right) v d s & =\int_{\Omega}\left(\varphi_{0}-\varphi^{\varepsilon}\right) \sigma\left(u^{\varepsilon}\right) \nabla \varphi^{\varepsilon} \nabla v d x \\
& +\int_{\Omega} \sigma\left(u^{\varepsilon}\right) v \nabla \varphi^{\varepsilon} \nabla \varphi_{0} d x \quad \forall v \in V_{D}(\Omega) \\
\int_{\Omega} \sigma\left(u^{\varepsilon}\right) \nabla \varphi^{\varepsilon} \nabla w d x & =0 \quad \varphi_{0}-\varphi^{\varepsilon} \in H_{0}^{1}(\Omega), \forall w \in H_{0}^{1}(\Omega) .
\end{aligned}
$$

Similarly, for $(u, \varphi)$ we have

$$
\begin{aligned}
\int_{\Omega} \nabla u \cdot \nabla \tilde{v} d x+\int_{\Gamma_{R}} \beta\left(u-u_{1}\right) \tilde{v} d s & =\int_{\Omega}\left(\varphi_{0}-\varphi\right) \sigma(u) \nabla \varphi \cdot \nabla \tilde{v} d x \\
& +\int_{\Omega} \sigma(u) \nabla \varphi \cdot \nabla \varphi_{0} \tilde{v} d x \quad \forall \tilde{v} \in V_{D}(\Omega), \\
\int_{\Omega} \sigma(u) \nabla \varphi \cdot \nabla \tilde{w} d x & =0 \quad \varphi-\varphi_{0} \in H_{0}^{1}(\Omega), \forall \tilde{w} \in H_{0}^{1}(\Omega) .
\end{aligned}
$$

Take the test functions $v=\left(u^{\varepsilon}-u\right) / \varepsilon, \tilde{v}=\left(u^{\varepsilon}-u\right) / \varepsilon, w=\left(\varphi^{\varepsilon}-\varphi\right) / \varepsilon$, and $\tilde{w}=\left(\varphi^{\varepsilon}-\varphi\right) / \varepsilon$, subtract corresponding equations in (5.4) from (5.3), and divide 
by $\varepsilon$ to obtain

$$
\begin{gathered}
\int_{\Omega} \nabla\left(\frac{u^{\varepsilon}-u}{\varepsilon}\right) \cdot \nabla\left(\frac{u^{\varepsilon}-u}{\varepsilon}\right) d x+\int_{\Gamma_{R}} \beta\left(\frac{u^{\varepsilon}-u}{\varepsilon}\right)\left(\frac{u^{\varepsilon}-u}{\varepsilon}\right) d s \\
=-\int_{\Gamma_{R}} \ell\left(u^{\varepsilon}-u_{1}\right)\left(\frac{u^{\varepsilon}-u}{\varepsilon}\right) d s \\
+\frac{1}{\varepsilon} \int_{\Omega}\left[\left(\varphi_{0}-\varphi^{\varepsilon}\right) \sigma\left(u^{\varepsilon}\right) \nabla \varphi^{\varepsilon}-\left(\varphi_{0}-\varphi\right) \sigma(u) \nabla \varphi\right] \cdot \nabla\left(\frac{u^{\varepsilon}-u}{\varepsilon}\right) d x \\
+\frac{1}{\varepsilon} \int_{\Omega}\left[\sigma\left(u^{\varepsilon}\right) \nabla \varphi^{\varepsilon}-\sigma(u) \nabla \varphi\right] \cdot \nabla \varphi_{0}\left(\frac{u^{\varepsilon}-u}{\varepsilon}\right) d x, \\
\frac{1}{\varepsilon} \int_{\Omega}\left[\sigma\left(u^{\varepsilon}\right) \nabla \varphi^{\varepsilon} \cdot \nabla\left(\frac{\varphi^{\varepsilon}-\varphi}{\varepsilon}\right)-\sigma(u) \nabla \varphi \cdot \nabla\left(\frac{\varphi^{\varepsilon}-\varphi}{\varepsilon}\right)\right] d x=0 .
\end{gathered}
$$

We derive $H^{1}(\Omega)$ estimate for $\left(\varphi^{\varepsilon}-\varphi\right) / \varepsilon$ first. Since $\left(\varphi^{\varepsilon}-\varphi\right) / \varepsilon \in H_{0}^{1}(\Omega)$ it follows from the Poincaré inequality that it is sufficient to have a bound on $\left\|\nabla\left(\varphi^{\varepsilon}-\varphi\right) / \varepsilon\right\|_{2}$.

The second equation in (5.5) implies

$$
\int_{\Omega} \sigma\left(u^{\varepsilon}\right) \nabla\left(\frac{\varphi^{\varepsilon}}{\varepsilon}\right) \cdot \nabla\left(\frac{\varphi^{\varepsilon}-\varphi}{\varepsilon}\right) d x=\int_{\Omega} \sigma(u) \nabla\left(\frac{\varphi}{\varepsilon}\right) \cdot \nabla\left(\frac{\varphi^{\varepsilon}-\varphi}{\varepsilon}\right) d x .
$$

Taking into account (5.6) we can write

$$
\begin{aligned}
& \int_{\Omega} \sigma(u)\left|\nabla\left(\frac{\varphi^{\varepsilon}-\varphi}{\varepsilon}\right)\right|^{2} d x=\int_{\Omega} \sigma(u) \nabla\left(\frac{\varphi^{\varepsilon}-\varphi}{\varepsilon}\right) \cdot \nabla\left(\frac{\varphi^{\varepsilon}-\varphi}{\varepsilon}\right) d x \\
& =\int_{\Omega} \sigma(u) \nabla\left(\frac{\varphi^{\varepsilon}}{\varepsilon}\right) \cdot \nabla\left(\frac{\varphi^{\varepsilon}-\varphi}{\varepsilon}\right) d x-\int_{\Omega} \sigma(u) \nabla\left(\frac{\varphi}{\varepsilon}\right) \cdot \nabla\left(\frac{\varphi^{\varepsilon}-\varphi}{\varepsilon}\right) d x \\
& =\int_{\Omega} \sigma(u) \nabla\left(\frac{\varphi^{\varepsilon}}{\varepsilon}\right) \cdot \nabla\left(\frac{\varphi^{\varepsilon}-\varphi}{\varepsilon}\right) d x-\int_{\Omega} \sigma\left(u^{\varepsilon}\right) \nabla\left(\frac{\varphi^{\varepsilon}}{\varepsilon}\right) \cdot \nabla\left(\frac{\varphi^{\varepsilon}-\varphi}{\varepsilon}\right) d x \\
& =\int_{\Omega}\left(\sigma(u)-\sigma\left(u^{\varepsilon}\right)\right) \nabla\left(\frac{\varphi^{\varepsilon}}{\varepsilon}\right) \cdot \nabla\left(\frac{\varphi^{\varepsilon}-\varphi}{\varepsilon}\right) d x .
\end{aligned}
$$

Remark 1. Observe that for a given weak solution $u$, it follows from Theorem 2.1. that if we set $C_{1}(u)=\sigma(N)$ with $N:=\|u\|_{\infty}$, then (since $0 \leq u \leq N<u_{*}$ ) we will have $0<C_{1}(u) \leq \sigma(u)$. Thus, we can write

$$
\begin{aligned}
& C_{1}(u) \int_{\Omega}\left|\nabla\left(\frac{\varphi^{\varepsilon}-\varphi}{\varepsilon}\right)\right|^{2} d x \leq \int_{\Omega} \sigma(u)\left|\nabla\left(\frac{\varphi^{\varepsilon}-\varphi}{\varepsilon}\right)\right|^{2} d x \\
& =\int_{\Omega}\left(\sigma(u)-\sigma\left(u^{\varepsilon}\right)\right) \nabla\left(\frac{\varphi^{\varepsilon}}{\varepsilon}\right) \cdot \nabla\left(\frac{\varphi^{\varepsilon}-\varphi}{\varepsilon}\right) d x \\
& \leq \int_{\Omega}\left|\frac{\sigma(u)-\sigma\left(u^{\varepsilon}\right)}{\varepsilon}\right| \cdot\left|\nabla \varphi^{\varepsilon}\right| \cdot\left|\nabla\left(\frac{\varphi^{\varepsilon}-\varphi}{\varepsilon}\right)\right| d x \\
& \leq K \int_{\Omega}\left|\frac{u^{\varepsilon}-u}{\varepsilon}\right| \cdot\left|\nabla \varphi^{\varepsilon}\right| \cdot\left|\nabla\left(\frac{\varphi^{\varepsilon}-\varphi}{\varepsilon}\right)\right| d x \\
& \leq K\left\|\frac{u^{\varepsilon}-u}{\varepsilon}\right\|_{s} \cdot\left\|\nabla \varphi^{\varepsilon}\right\|_{r} \cdot\left\|\nabla\left(\frac{\varphi^{\varepsilon}-\varphi}{\varepsilon}\right)\right\|_{2},
\end{aligned}
$$

where we used (5.7). Thus, $C_{1}(u)\left\|\nabla\left(\frac{\varphi^{\varepsilon}-\varphi}{\varepsilon}\right)\right\|_{2}^{2} \leq K\left\|\frac{u^{\varepsilon}-u}{\varepsilon}\right\|_{s} \cdot\left\|\nabla \varphi^{\varepsilon}\right\|_{r} \cdot\left\|\nabla\left(\frac{\varphi^{\varepsilon}-\varphi}{\varepsilon}\right)\right\|_{2}$ and therefore

$$
\left\|\nabla\left(\frac{\varphi^{\varepsilon}-\varphi}{\varepsilon}\right)\right\|_{2} \leq \frac{K}{C_{1}(u)}\left\|\frac{u^{\varepsilon}-u}{\varepsilon}\right\|_{s} \cdot\left\|\nabla \varphi^{\varepsilon}\right\|_{r} .
$$


Since $H^{1}(\Omega) \subset L^{s}(\Omega)$ we have

$$
\left\|\frac{u^{\varepsilon}-u}{\varepsilon}\right\|_{s} \leq M_{1}\left\|\frac{u^{\varepsilon}-u}{\varepsilon}\right\|_{H^{1}(\Omega)}
$$

By Theorem 3.1 we have $\left\|\nabla \varphi^{\varepsilon}\right\|_{r} \leq \Phi$. Substituting this estimate and (5.10) into (5.9) we get

$$
\left\|\nabla\left(\frac{\varphi^{\varepsilon}-\varphi}{\varepsilon}\right)\right\|_{2} \leq \frac{K M_{1} \Phi}{C_{1}(u)}\left\|\frac{u^{\varepsilon}-u}{\varepsilon}\right\|_{H^{1}(\Omega)} .
$$

Using the Poincaré inequality, we obtain

$$
\left\|\frac{\varphi^{\varepsilon}-\varphi}{\varepsilon}\right\|_{H^{1}(\Omega)} \leq C_{6}\left\|\nabla\left(\frac{\varphi^{\varepsilon}-\varphi}{\varepsilon}\right)\right\|_{2} \leq \frac{C_{6} K M_{1} \Phi}{C_{1}(u)}\left\|\frac{u^{\varepsilon}-u}{\varepsilon}\right\|_{H^{1}(\Omega)} .
$$

Now we proceed to estimate the $H^{1}$ norm of $\left(u^{\varepsilon}-u\right) / \varepsilon$. We obtain from (5.5)

$$
\begin{aligned}
& \int_{\Omega}\left|\nabla\left(\frac{u^{\varepsilon}-u}{\varepsilon}\right)\right|^{2} d x \leq \int_{\Omega}\left|\nabla\left(\frac{u^{\varepsilon}-u}{\varepsilon}\right)\right|^{2} d x+\int_{\Gamma_{R}} \beta\left(\frac{u^{\varepsilon}-u}{\varepsilon}\right)^{2} d s \\
& =\left|-\int_{\Gamma_{R}} \ell\left(u^{\varepsilon}-u_{1}\right)\left(\frac{u^{\varepsilon}-u}{\varepsilon}\right) d s+\mathcal{C}+\mathcal{D}\right|,
\end{aligned}
$$

where $\mathcal{C} \stackrel{\text { def }}{=} \frac{1}{\varepsilon} \int_{\Omega}\left[\left(\varphi_{0}-\varphi^{\varepsilon}\right) \sigma\left(u^{\varepsilon}\right) \nabla \varphi^{\varepsilon}-\left(\varphi_{0}-\varphi\right) \sigma(u) \nabla \varphi\right] \cdot \nabla\left(\frac{u^{\varepsilon}-u}{\varepsilon}\right) d x=\int_{\Omega}\left[\left(\varphi_{0}-\right.\right.$ $\left.\varphi)\left(\frac{\sigma\left(u^{\varepsilon}\right) \nabla \varphi^{\varepsilon}-\sigma(u) \nabla \varphi}{\varepsilon}\right)+\left(\frac{\varphi-\varphi^{\varepsilon}}{\varepsilon}\right) \sigma\left(u^{\varepsilon}\right) \nabla \varphi^{\varepsilon}\right] \cdot \nabla\left(\frac{u^{\varepsilon}-u}{\varepsilon}\right) d x$ and $\mathcal{D} \stackrel{\text { def }}{=} \frac{1}{\varepsilon} \int_{\Omega}\left[\sigma\left(u^{\varepsilon}\right) \nabla \varphi^{\varepsilon}-\right.$ $\sigma(u) \nabla \varphi] \cdot \nabla \varphi_{0}\left(\frac{u^{\varepsilon}-u}{\varepsilon}\right) d x$. First, we estimate $|\mathcal{C}|$ to get

$$
\begin{aligned}
|\mathcal{C}| & \leq \int_{\Omega}\left|\varphi_{0}-\varphi\right| \cdot\left|\frac{\sigma\left(u^{\varepsilon}\right) \nabla \varphi^{\varepsilon}-\sigma(u) \nabla \varphi}{\varepsilon}\right| \cdot\left|\nabla\left(\frac{u^{\varepsilon}-u}{\varepsilon}\right)\right| d x \\
& +\int_{\Omega}\left|\frac{\varphi-\varphi^{\varepsilon}}{\varepsilon}\right| \sigma\left(u^{\varepsilon}\right)\left|\nabla \varphi^{\varepsilon}\right| \cdot\left|\nabla\left(\frac{u^{\varepsilon}-u}{\varepsilon}\right)\right| d x \\
& \leq 2 \tilde{M} \int_{\Omega}\left|\frac{\sigma\left(u^{\varepsilon}\right) \nabla \varphi^{\varepsilon}-\sigma(u) \nabla \varphi}{\varepsilon}\right| \cdot\left|\nabla\left(\frac{u^{\varepsilon}-u}{\varepsilon}\right)\right| d x \\
& +\mu \int_{\Omega}\left|\frac{\varphi-\varphi^{\varepsilon}}{\varepsilon}\right| \cdot\left|\nabla \varphi^{\varepsilon}\right| \cdot\left|\nabla\left(\frac{u^{\varepsilon}-u}{\varepsilon}\right)\right| d x \\
& =2 \tilde{M} \int_{\Omega}\left|\frac{\left(\sigma\left(u^{\varepsilon}\right)-\sigma(u)+\sigma(u)\right) \nabla \varphi^{\varepsilon}-\sigma(u) \nabla \varphi}{\varepsilon}\right| \cdot\left|\nabla\left(\frac{u^{\varepsilon}-u}{\varepsilon}\right)\right| d x \\
& +\mu \int_{\Omega}\left|\frac{\varphi-\varphi^{\varepsilon}}{\varepsilon}\right| \cdot\left|\nabla \varphi^{\varepsilon}\right| \cdot\left|\nabla\left(\frac{u^{\varepsilon}-u}{\varepsilon}\right)\right| d x \\
& \leq 2 \tilde{M} \int_{\Omega}\left|\frac{\sigma\left(u^{\varepsilon}\right)-\sigma(u)}{\varepsilon}\right| \cdot\left|\nabla \varphi^{\varepsilon}\right| \cdot\left|\nabla\left(\frac{u^{\varepsilon}-u}{\varepsilon}\right)\right| d x \\
& +2 \tilde{M} \int_{\Omega} \sigma(u) \cdot\left|\nabla\left(\frac{\varphi^{\varepsilon}-\varphi}{\varepsilon}\right)\right| \cdot\left|\nabla\left(\frac{u^{\varepsilon}-u}{\varepsilon}\right)\right| d x \\
& +\mu \int_{\Omega}\left|\frac{\varphi-\varphi^{\varepsilon}}{\varepsilon}\right| \cdot\left|\nabla \varphi^{\varepsilon}\right| \cdot\left|\nabla\left(\frac{u^{\varepsilon}-u}{\varepsilon}\right)\right| d x \\
& \leq 2 \tilde{M} K \int_{\Omega}\left|\frac{u^{\varepsilon}-u}{\varepsilon}\right| \cdot\left|\nabla \varphi^{\varepsilon}\right| \cdot\left|\nabla\left(\frac{u^{\varepsilon}-u}{\varepsilon}\right)\right| d x \\
& +2 \tilde{M} \mu \int_{\Omega}\left|\nabla\left(\frac{\varphi^{\varepsilon}-\varphi}{\varepsilon}\right)\right| \cdot\left|\nabla\left(\frac{u^{\varepsilon}-u}{\varepsilon}\right)\right| d x \\
& +\mu \int_{\Omega}\left|\frac{\varphi-\varphi^{\varepsilon}}{\varepsilon}\right| \cdot\left|\nabla \varphi^{\varepsilon}\right| \cdot\left|\nabla\left(\frac{u^{\varepsilon}-u}{\varepsilon}\right)\right| d x .
\end{aligned}
$$


Next, estimating $|\mathcal{D}|$ we obtain

$$
\begin{aligned}
|\mathcal{D}| & \leq \int_{\Omega}\left|\nabla \varphi_{0}\right| \cdot\left|\frac{\sigma\left(u^{\varepsilon}\right)-\sigma(u)}{\varepsilon}\right| \cdot\left|\nabla \varphi^{\varepsilon}\right| \cdot\left|\frac{u^{\varepsilon}-u}{\varepsilon}\right| d x \\
& +\int_{\Omega} \sigma(u)\left|\nabla \varphi_{0}\right| \cdot\left|\nabla\left(\frac{\varphi^{\varepsilon}-\varphi}{\varepsilon}\right)\right| \cdot\left|\frac{u^{\varepsilon}-u}{\varepsilon}\right| d x \\
& \leq K\left\|\nabla \varphi_{0}\right\|_{\infty} \int_{\Omega}\left|\frac{u^{\varepsilon}-u}{\varepsilon}\right| \cdot\left|\nabla \varphi^{\varepsilon}\right| \cdot\left|\frac{u^{\varepsilon}-u}{\varepsilon}\right| d x \\
& +\mu\left\|\nabla \varphi_{0}\right\|_{\infty} \int_{\Omega}\left|\nabla\left(\frac{\varphi^{\varepsilon}-\varphi}{\varepsilon}\right)\right| \cdot\left|\frac{u^{\varepsilon}-u}{\varepsilon}\right| d x .
\end{aligned}
$$

Thus we have

$$
\begin{aligned}
& \int_{\Omega}\left|\nabla\left(\frac{u^{\varepsilon}-u}{\varepsilon}\right)\right|^{2} d x \leq \int_{\Gamma_{R}}|\ell| \cdot\left|u^{\varepsilon}-u_{1}\right| \cdot\left|\frac{u^{\varepsilon}-u}{\varepsilon}\right| d s+|\mathcal{C}|+|\mathcal{D}| \\
& \leq \mathcal{M} \int_{\Gamma_{R}}\left|u^{\varepsilon}-u_{1}\right| \cdot\left|\frac{u^{\varepsilon}-u}{\varepsilon}\right| d s+|\mathcal{C}|+|\mathcal{D}| \\
& \leq \mathcal{M}\left\|u^{\varepsilon}-u_{1}\right\|_{L^{2}\left(\Gamma_{R}\right)} \cdot\left\|\frac{u^{\varepsilon}-u}{\varepsilon}\right\|_{L^{2}(\partial \Omega)}+|\mathcal{C}|+|\mathcal{D}| \\
& \leq \mathcal{M} M_{2}^{2}\left\|u^{\varepsilon}-u_{1}\right\|_{H^{1}(\Omega)} \cdot\left\|\frac{u^{\varepsilon}-u}{\varepsilon}\right\|_{H^{1}(\Omega)}+|\mathcal{C}|+|\mathcal{D}|
\end{aligned}
$$

where we have used the trace inequality $\left\|u^{\varepsilon}-u_{1}\right\|_{L^{2}(\partial \Omega)} \leq M_{2}\left\|u^{\varepsilon}\right\|_{H^{1}(\Omega)}$. Taking into account the estimates for $|\mathcal{C}|$ and $|\mathcal{D}|$ we get

$$
\begin{aligned}
& \int_{\Omega}\left|\nabla\left(\frac{u^{\varepsilon}-u}{\varepsilon}\right)\right|^{2} d x \leq \mathcal{M} M_{2}^{2}\left\|u^{\varepsilon}-u_{1}\right\|_{H^{1}(\Omega)} \cdot\left\|\frac{u^{\varepsilon}-u}{\varepsilon}\right\|_{H^{1}(\Omega)} \\
& +2 \tilde{M} K \int_{\Omega}\left|\frac{u^{\varepsilon}-u}{\varepsilon}\right| \cdot\left|\nabla \varphi^{\varepsilon}\right| \cdot\left|\nabla\left(\frac{u^{\varepsilon}-u}{\varepsilon}\right)\right| d x+2 \tilde{M} \mu \int_{\Omega}\left|\nabla\left(\frac{\varphi^{\varepsilon}-\varphi}{\varepsilon}\right)\right| \cdot\left|\nabla\left(\frac{u^{\varepsilon}-u}{\varepsilon}\right)\right| d x \\
& +\mu \int_{\Omega}\left|\frac{\varphi-\varphi^{\varepsilon}}{\varepsilon}\right| \cdot\left|\nabla \varphi^{\varepsilon}\right| \cdot\left|\nabla\left(\frac{u^{\varepsilon}-u}{\varepsilon}\right)\right| d x+K\left\|\nabla \varphi_{0}\right\|_{\infty} \int_{\Omega}\left|\frac{u^{\varepsilon}-u}{\varepsilon}\right| \cdot\left|\nabla \varphi^{\varepsilon}\right| \cdot\left|\frac{u^{\varepsilon}-u}{\varepsilon}\right| d x \\
& +\mu\left\|\nabla \varphi_{0}\right\|_{\infty} \int_{\Omega}\left|\nabla\left(\frac{\varphi^{\varepsilon}-\varphi}{\varepsilon}\right)\right| \cdot\left|\frac{u^{\varepsilon}-u}{\varepsilon}\right| d x .
\end{aligned}
$$

Using the Hölder inequality, and a priori bounds (3.1), (5.10), and (5.12) we have

$$
\begin{aligned}
& \int_{\Omega}\left|\nabla\left(\frac{u^{\varepsilon}-u}{\varepsilon}\right)\right|^{2} d x \\
& \leq \mathcal{M} M_{2}^{2} \tilde{C}\left\|\frac{u^{\varepsilon}-u}{\varepsilon}\right\|_{H^{1}(\Omega)} \\
& +2 \tilde{M} K\left\|\frac{u^{\varepsilon}-u}{\varepsilon}\right\|_{s} \cdot\left\|\nabla \varphi^{\varepsilon}\right\|_{r} \cdot\left\|\nabla\left(\frac{u^{\varepsilon}-u}{\varepsilon}\right)\right\|_{2} \\
& +2 \tilde{M} \mu\left\|\nabla\left(\frac{\varphi^{\varepsilon}-\varphi}{\varepsilon}\right)\right\|_{2} \cdot\left\|\nabla\left(\frac{u^{\varepsilon}-u}{\varepsilon}\right)\right\|_{2} \\
& +\mu\left\|\frac{\varphi^{\varepsilon}-\varphi}{\varepsilon}\right\|_{s} \cdot\left\|\nabla \varphi^{\varepsilon}\right\|_{r} \cdot\left\|\nabla\left(\frac{u^{\varepsilon}-u}{\varepsilon}\right)\right\|_{2} \\
& +K\left\|\nabla \varphi_{0}\right\|_{\infty} \cdot\left\|\frac{u^{\varepsilon}-u}{\varepsilon}\right\|_{s} \cdot\left\|\nabla \varphi^{\varepsilon}\right\|_{r} \cdot\left\|\frac{u^{\varepsilon}-u}{\varepsilon}\right\|_{2} \\
& +\mu\left\|\nabla \varphi_{0}\right\|_{\infty} \cdot\left\|\nabla\left(\frac{\varphi^{\varepsilon}-\varphi}{\varepsilon}\right)\right\|_{2} \cdot\left\|\frac{u^{\varepsilon}-u}{\varepsilon}\right\|_{2}
\end{aligned}
$$




$$
\begin{aligned}
& \leq \mathcal{M} M_{2}^{2} \tilde{C}\left\|\frac{u^{\varepsilon}-u}{\varepsilon}\right\|_{H^{1}(\Omega)}+2 \tilde{M} K M_{1} \Phi\left\|\frac{u^{\varepsilon}-u}{\varepsilon}\right\|_{H^{1}(\Omega)}^{2} \\
& +\frac{2 \tilde{M} \mu K M_{1} \Phi}{C_{1}(u)}\left\|\frac{u^{\varepsilon}-u}{\varepsilon}\right\|_{H^{1}(\Omega)}^{2}+\frac{\mu C_{6} K M_{1} \Phi^{2}}{C_{1}(u)}\left\|\frac{u^{\varepsilon}-u}{\varepsilon}\right\|_{H^{1}(\Omega)}^{2} \\
& +K\left\|\nabla \varphi_{0}\right\|_{\infty} M_{1} \Phi\left\|\frac{u^{\varepsilon}-u}{\varepsilon}\right\|_{H^{1}(\Omega)}^{2}+\mu\left\|\nabla \varphi_{0}\right\|_{\infty} \frac{K M_{1} \Phi}{C_{1}(u)}\left\|\frac{u^{\varepsilon}-u}{\varepsilon}\right\|_{H^{1}(\Omega)}^{2} .
\end{aligned}
$$

Since $u-u^{\varepsilon} \in V_{D}$, it follows from the extension of the Poincaré inequality (see Theorem 5.8 in 20]) that there exists $k>0$ such that

$$
\begin{aligned}
& k\left\|\frac{u^{\varepsilon}-u}{\varepsilon}\right\|_{H^{1}(\Omega)}^{2} \leq \int_{\Omega}\left|\nabla\left(\frac{u^{\varepsilon}-u}{\varepsilon}\right)\right|^{2} d x \leq \mathcal{M} M_{2}^{2} \tilde{C}\left\|\frac{u^{\varepsilon}-u}{\varepsilon}\right\|_{H^{1}(\Omega)} \\
& +2 \tilde{M} K M_{1} \Phi\left\|\frac{u^{\varepsilon}-u}{\varepsilon}\right\|_{H^{1}(\Omega)}^{2}+\frac{2 \tilde{M} \mu K M_{1} \Phi}{C_{1}(u)}\left\|\frac{u^{\varepsilon}-u}{\varepsilon}\right\|_{H^{1}(\Omega)}^{2}+\frac{\mu C_{6} K M_{1} \Phi^{2}}{C_{1}(u)}\left\|\frac{u^{\varepsilon}-u}{\varepsilon}\right\|_{H^{1}(\Omega)}^{2} \\
& +K\left\|\nabla \varphi_{0}\right\|_{\infty} M_{1} \Phi\left\|\frac{u^{\varepsilon}-u}{\varepsilon}\right\|_{H^{1}(\Omega)}^{2}+\mu\left\|\nabla \varphi_{0}\right\|_{\infty} \frac{K M_{1} \Phi}{C_{1}(u)}\left\|\frac{u^{\varepsilon}-u}{\varepsilon}\right\|_{H^{1}(\Omega)}^{2} .
\end{aligned}
$$

By definition, $\Phi$ includes $\left\|\nabla \varphi_{0}\right\|_{\infty}$ and $\left\|\varphi_{0}\right\|_{W^{1, \infty}(\Omega)}$. Hence, if $\left\|\varphi_{0}\right\|_{W^{1, \infty}(\Omega)}$ is chosen small enough so that

$$
\begin{gathered}
k_{1} \equiv k-2 \tilde{M} K M_{1} \Phi-\frac{2 \tilde{M} \mu K M_{1} \Phi}{C_{1}(u)}-\frac{\mu C_{6} K M_{1} \Phi^{2}}{C_{1}(u)} \\
-K\left\|\nabla \varphi_{0}\right\|_{\infty} M_{1} \Phi-\mu\left\|\nabla \varphi_{0}\right\|_{\infty} \frac{K M_{1} \Phi}{C_{1}(u)}>0
\end{gathered}
$$

then

$$
\left\|\frac{u^{\varepsilon}-u}{\varepsilon}\right\|_{H^{1}(\Omega)} \leq \frac{\mathcal{M} M_{2}^{2} \tilde{C}}{k_{1}},
$$

where the constant in (5.14) does not depend on $\varepsilon$. Consequently, (5.12) yields

$$
\left\|\frac{\varphi^{\varepsilon}-\varphi}{\varepsilon}\right\|_{H^{1}(\Omega)} \leq \frac{C_{6} K M_{1} \Phi}{C_{1}(u)}\left\|\frac{u^{\varepsilon}-u}{\varepsilon}\right\|_{H^{1}(\Omega)} \leq \frac{C_{6} K M_{1} \Phi}{C_{1}(u)} \cdot \frac{\mathcal{M} M_{2}^{2} \tilde{C}}{k_{1}} .
$$

These estimates justify the existence of $\psi_{1}$ and $\psi_{2}$, and the convergences in (5.1). All in all, we have the following convergences

$$
\begin{aligned}
& \frac{u^{\varepsilon}-u}{\varepsilon} \stackrel{w}{\rightarrow} \psi_{1} \text { in } H^{1}(\Omega), \frac{u^{\varepsilon}-u}{\varepsilon} \stackrel{s}{\rightarrow} \psi_{1} \text { in } L^{s}(\Omega), \\
& \frac{\varphi^{\varepsilon}-\varphi}{\varepsilon} \stackrel{w}{\rightarrow} \psi_{2} \text { in } H^{1}(\Omega), \frac{\varphi^{\varepsilon}-\varphi}{\varepsilon} \stackrel{s}{\rightarrow} \psi_{2} \text { in } L^{s}(\Omega), \\
& u^{\varepsilon} \stackrel{s}{\rightarrow} u \text { in } L^{s}(\Omega), \varphi^{\varepsilon} \stackrel{s}{\rightarrow} \varphi \text { in } C(\bar{\Omega}), \\
& \nabla \varphi^{\varepsilon} \stackrel{w}{\rightarrow} \nabla \varphi \text { in } L^{r}(\Omega), \frac{u^{\varepsilon}-u}{\varepsilon} \stackrel{w}{\rightarrow} \psi_{1} \text { in } L^{2}\left(\Gamma_{R}\right), \\
& \beta^{\varepsilon} \stackrel{w}{\rightarrow} \beta \text { in } L^{2}\left(\Gamma_{R}\right), \beta^{\varepsilon} \stackrel{w *}{\rightarrow} \beta \text { in } L^{\infty}\left(\Gamma_{R}\right) \text { as } \varepsilon \rightarrow 0,
\end{aligned}
$$

and therefore, we can show that the sensitivities satisfy the system (5.2).

Remark 2. At this point the convergences are on a subsequence and in order to obtain the desired convergence for the whole sequence, it needs to be shown that 
the limits $\psi_{1}$ and $\psi_{2}$ are always the same for any subsequence. This uniqueness of the limits follows from (5.2) (since the system in $\psi_{1}$ and $\psi_{2}$ is non-degenerate linear elliptic) which $\psi_{1}$ and $\psi_{2}$ produced by subsequences will necessarily satisfy.

Subtracting (5.4) from (5.3), and dividing by $\varepsilon$ :

$$
\begin{aligned}
& \int_{\Omega} \nabla\left(\frac{u^{\varepsilon}-u}{\varepsilon}\right) \cdot \nabla v d x+\int_{\Gamma_{R}} \beta\left(\frac{u^{\varepsilon}-u}{\varepsilon}\right) v d s+\int_{\Gamma_{R}} \ell\left(u^{\varepsilon}-u_{1}\right) v d s \\
= & \frac{1}{\varepsilon} \int_{\Omega}\left[\left(\varphi_{0}-\varphi^{\varepsilon}\right) \sigma\left(u^{\varepsilon}\right) \nabla \varphi^{\varepsilon} \cdot \nabla v-\left(\varphi_{0}-\varphi\right) \sigma(u) \nabla \varphi \cdot \nabla v\right] d x \\
+ & \frac{1}{\varepsilon} \int_{\Omega}\left[\sigma\left(u^{\varepsilon}\right) \nabla \varphi^{\varepsilon}-\sigma(u) \nabla \varphi\right] \cdot \nabla \varphi_{0} v d x \quad \forall v \in V_{D}(\Omega), \\
& \frac{1}{\varepsilon} \int_{\Omega}\left[\sigma\left(u^{\varepsilon}\right) \nabla \varphi^{\varepsilon} \cdot \nabla w-\sigma(u) \nabla \varphi \cdot \nabla w\right] d x=0 \quad \forall w \in H_{0}^{1}(\Omega) .
\end{aligned}
$$

The convergence proofs for various terms are rather standard if sometimes lengthy. For example, it can be shown easily that the terms on the left hand side of (5.16) converge because of the weak convergence of the corresponding sequences. We omit the details. The first term on the right hand side of (5.16) can be written as follows

$$
\begin{aligned}
& \frac{1}{\varepsilon} \int_{\Omega}\left[\left(\varphi_{0}-\varphi^{\varepsilon}\right) \sigma\left(u^{\varepsilon}\right) \nabla \varphi^{\varepsilon} \nabla v-\left(\varphi_{0}-\varphi\right) \sigma(u) \nabla \varphi \nabla v\right] d x \\
= & \frac{1}{\varepsilon} \int_{\Omega}\left(\varphi_{0}-\varphi^{\varepsilon}\right)\left[\sigma\left(u^{\varepsilon}\right) \nabla \varphi^{\varepsilon}-\sigma(u) \nabla \varphi\right] \nabla v d x+\frac{1}{\varepsilon} \int_{\Omega}\left(\varphi-\varphi^{\varepsilon}\right) \sigma(u) \nabla \varphi \nabla v d x \\
= & \frac{1}{\varepsilon} \int_{\Omega}\left(\varphi_{0}-\varphi^{\varepsilon}\right)\left[\sigma\left(u^{\varepsilon}\right)-\sigma(u)\right] \nabla \varphi^{\varepsilon} \nabla v d x+\frac{1}{\varepsilon} \int_{\Omega}\left(\varphi_{0}-\varphi^{\varepsilon}\right) \sigma(u)\left[\nabla \varphi^{\varepsilon}-\nabla \varphi\right] \nabla v d x \\
+ & \frac{1}{\varepsilon} \int_{\Omega}\left(\varphi-\varphi^{\varepsilon}\right) \sigma(u) \nabla \varphi \nabla v d x=\mathcal{G}_{1}+\mathcal{G}_{2}+\mathcal{F} .
\end{aligned}
$$

A detailed derivation for the convergence of $\mathcal{G}_{1}$ can be found in 14. Therefore, for the sake of completeness, we show the convergence of $\mathcal{G}_{2}$ and $\mathcal{F}$ here. First, we need to show that

$$
\mathcal{F} \rightarrow-\int_{\Omega} \psi_{2} \sigma(u) \nabla \varphi \nabla v d x \text { as } \varepsilon \rightarrow 0 .
$$

Indeed, we can write

$$
\begin{aligned}
& \left|\int_{\Omega}\left(\frac{\varphi-\varphi^{\varepsilon}}{\varepsilon}\right) \sigma(u) \nabla \varphi \nabla v d x-\int_{\Omega}\left(-\psi_{2}\right) \sigma(u) \nabla \varphi \nabla v d x\right| \\
& \leq C_{2} \int_{\Omega}\left|\frac{\varphi^{\varepsilon}-\varphi}{\varepsilon}-\psi_{2}\right||\varphi||\nabla v| d x \leq C_{2}\left\|\frac{\varphi^{\varepsilon}-\varphi}{\varepsilon}-\psi_{2}\right\|_{s}\|\nabla \varphi\|_{r}\|\nabla v\|_{2} \rightarrow 0 \text { as } \varepsilon \rightarrow 0 .
\end{aligned}
$$

This proves (5.18). Next, we show that

$$
\mathcal{G}_{2} \rightarrow \int_{\Omega}\left(\varphi_{0}-\varphi\right) \sigma(u) \nabla \psi_{2} \nabla v d x \text { as } \varepsilon \rightarrow 0
$$

We write

$$
\begin{aligned}
\mathcal{G}_{2} & =\frac{1}{\varepsilon} \int_{\Omega}\left(\varphi_{0}-\varphi\right) \sigma(u) \nabla\left(\varphi^{\varepsilon}-\varphi\right) \nabla v d x \\
& +\frac{1}{\varepsilon} \int_{\Omega}\left(\varphi-\varphi^{\varepsilon}\right) \sigma(u) \nabla\left(\varphi^{\varepsilon}-\varphi\right) \nabla v d x=\mathcal{G}_{21}+\mathcal{G}_{22}
\end{aligned}
$$


For the term $\mathcal{G}_{21}$ we have

$$
\begin{aligned}
& \left|\mathcal{G}_{21}-\int_{\Omega}\left(\varphi_{0}-\varphi\right) \sigma(u) \nabla \psi_{2} \cdot \nabla v d x\right| \\
& =\mid \int_{\Omega}\left[\left(\varphi_{0}-\varphi\right) \sigma(u) \nabla\left(\frac{\varphi^{\varepsilon}-\varphi}{\varepsilon}\right) \cdot \nabla v-\left(\varphi_{0}-\varphi\right) \sigma(u) \nabla \psi_{2} \cdot \nabla v\right] d x \\
& =\left|\int_{\Omega}\left[\nabla\left(\frac{\varphi^{\varepsilon}-\varphi}{\varepsilon}\right)-\nabla \psi_{2}\right]\left(\varphi_{0}-\varphi\right) \sigma(u) \nabla v d x\right| \rightarrow 0 \quad \text { as } \varepsilon \rightarrow 0
\end{aligned}
$$

since $\nabla\left(\varphi^{\varepsilon}-\varphi\right) / \varepsilon \stackrel{w}{\longrightarrow} \nabla \psi_{2}$ in $L^{2}(\Omega)$ and $\left(\varphi_{0}-\varphi\right) \sigma(u) \nabla v \in L^{2}(\Omega)$ because $r>d$ implies $\varphi_{0}-\varphi \in W^{1, r}(\Omega) \subset \subset C(\bar{\Omega})$. Now we show the convergence of $\mathcal{G}_{22}$. Indeed, we have

$$
\begin{aligned}
\left|\mathcal{G}_{22}\right| & =\left|\int_{\Omega}\left(\varphi-\varphi^{\varepsilon}\right) \sigma(u) \nabla\left(\frac{\varphi^{\varepsilon}-\varphi}{\varepsilon}\right) \cdot \nabla v d x\right| \\
& \leq C_{2} \int_{\Omega}\left|\varphi-\varphi^{\varepsilon}\right| \cdot\left|\nabla\left(\frac{\varphi^{\varepsilon}-\varphi}{\varepsilon}\right)\right| \cdot|\nabla v| d x \\
& \leq C_{2}\left\|\varphi-\varphi^{\varepsilon}\right\|_{C(\bar{\Omega})} \cdot\left\|\nabla\left(\frac{\varphi^{\varepsilon}-\varphi}{\varepsilon}\right)\right\|_{2} \cdot\|\nabla v\|_{2} \rightarrow 0 \quad \text { as } \varepsilon \rightarrow 0
\end{aligned}
$$

since $\left\|\varphi-\varphi^{\varepsilon}\right\|_{C(\bar{\Omega})} \rightarrow 0$ as $\varepsilon \rightarrow 0$, and $\left.\| \nabla\left(\varphi^{\varepsilon}-\varphi\right) / \varepsilon\right) \|_{2}$ is bounded. This ends the proof of (5.19). Similarly, it can be shown for the second term on the right hand side of (5.16) that:

$$
\begin{aligned}
\frac{1}{\varepsilon} \int_{\Omega}\left[\sigma\left(u^{\varepsilon}\right) \nabla \varphi^{\varepsilon}-\sigma(u) \nabla \varphi\right] \cdot \nabla \varphi_{0} v d x & \rightarrow \int_{\Omega} \sigma^{\prime}(u) \psi_{1} \nabla \varphi \cdot \nabla \varphi_{0} v d x \\
& +\int_{\Omega} \sigma(u) \nabla \psi_{2} \cdot \nabla \varphi_{0} v d x \text { as } \varepsilon \rightarrow 0 .
\end{aligned}
$$

Finally, the terms of (5.17) satisfy

$$
\begin{aligned}
\frac{1}{\varepsilon} \int_{\Omega}\left[\sigma\left(u^{\varepsilon}\right) \nabla \varphi^{\varepsilon} \cdot \nabla w-\sigma(u) \nabla \varphi \cdot \nabla w\right] d x & \rightarrow \int_{\Omega} \sigma^{\prime}(u) \psi_{1} \nabla \varphi \cdot \nabla w d x \\
& +\int_{\Omega} \sigma(u) \nabla \psi_{2} \cdot \nabla w d x \text { as } \varepsilon \rightarrow 0 .
\end{aligned}
$$

Letting $\varepsilon \rightarrow 0$ in (5.16) and (5.17) we obtain

$$
\begin{aligned}
& \int_{\Omega} \nabla \psi_{1} \nabla v d x+\int_{\Gamma_{R}}\left(\beta \psi_{1}+\ell\left(u-u_{1}\right)\right) v d s=\int_{\Omega}\left(\varphi_{0}-\varphi\right) \sigma^{\prime}(u) \psi_{1} \nabla \varphi \nabla v d x \\
+ & \int_{\Omega}\left(\varphi_{0}-\varphi\right) \sigma(u) \nabla \psi_{2} \nabla v d x-\int_{\Omega} \psi_{2} \sigma(u) \nabla \varphi \nabla v d x \\
+ & \int_{\Omega} \sigma^{\prime}(u) \psi_{1} \nabla \varphi \nabla \varphi_{0} v d x+\int_{\Omega} \sigma(u) \nabla \psi_{2} \nabla \varphi_{0} v d x \quad \forall v \in V_{D}(\Omega) \\
& \int_{\Omega} \sigma^{\prime}(u) \psi_{1} \nabla \varphi \nabla w+\int_{\Omega} \sigma(u) \nabla \psi_{2} \nabla w d x=0 \quad \forall w \in H_{0}^{1}(\Omega) .
\end{aligned}
$$


It can shown that the "strong" formulation corresponding to (5.21) is given by

$$
\begin{aligned}
\Delta \psi_{1}+\sigma^{\prime}(u) \psi_{1}|\nabla \varphi|^{2}+2 \sigma(u) \nabla \varphi \cdot \nabla \psi_{2} & =0 \text { in } \Omega, \\
\nabla \cdot\left(\sigma^{\prime}(u) \psi_{1} \nabla \varphi+\sigma(u) \nabla \psi_{2}\right) & =0 \text { in } \Omega, \\
\frac{\partial \psi_{1}}{\partial n}+\beta \psi_{1}+\ell\left(u-u_{1}\right) & =0 \text { on } \Gamma_{R}, \\
\psi_{1} & =0 \text { on } \Gamma_{D}, \\
\psi_{2} & =0 \text { on } \partial \Omega .
\end{aligned}
$$

$\square$

In order to characterize the optimal control, we need to introduce adjoint functions $p$ and $q$ as well as the adjoint operator associated with $\psi_{1}$ and $\psi_{2}$. Using the same reasoning as in [14, it can be shown that the adjoint system is given by

$$
\begin{aligned}
\Delta p+\sigma^{\prime}(u)|\nabla \varphi|^{2} p-\sigma^{\prime}(u) \nabla \varphi \cdot \nabla q & =1 \text { in } \Omega, \\
\nabla \cdot[-2 p \sigma(u) \nabla \varphi+\sigma(u) \nabla q] & =0 \text { in } \Omega, \\
\frac{\partial p}{\partial n}+\beta^{*} p & =0 \text { on } \Gamma_{R}, \\
p & =0 \text { on } \Gamma_{D}, \\
q & =0 \text { on } \partial \Omega,
\end{aligned}
$$

where the nonhomogeneous term "1" comes from differentiating the integrand of $J(\beta)$ with respect to the state $u$.

THEOREM 5.2. Let $\left\|\varphi_{0}\right\|_{W^{1, \infty}(\Omega)}$ be sufficiently small. Then, given an optimal control $\beta^{*} \in U_{\mathcal{M}}$ and the corresponding states $u, \varphi$, there exists a solution $(p, q) \in H^{1}(\Omega) \times H_{0}^{1}(\Omega)$ to the adjoint system (5.23). Furthermore, $\beta^{*}$ can be explicitly characterized as:

$$
\beta^{*}(x)=\min \left(\max \left(-\frac{\left(u-u_{1}\right) p}{2}, 0\right), \mathcal{M}\right) .
$$

Proof. Observe that the existence of solution to the adjoint system (5.23) can be proved using Banach fixed point theorem in a similar way to how it was done in [14]. Now we consider the derivation of the characterization of the optimal control. For a variation $\ell \in L^{\infty}\left(\Gamma_{R}\right)$, with $\beta^{*}+\varepsilon \ell \in U_{\mathcal{M}}$, the weak formulation of the sensitivity system (5.2) is given by

$$
\begin{gathered}
-\int_{\Omega} \nabla \psi_{1} \cdot \nabla v d x-\int_{\Gamma_{R}} \beta \psi_{1} v d s+\int_{\Omega} \sigma^{\prime}(u) \psi_{1}|\nabla \varphi|^{2} v d x \\
+2 \int_{\Omega} \sigma(u) \nabla \varphi \cdot \nabla \psi_{2} v d x=\int_{\Gamma_{R}} \ell\left(u-u_{1}\right) v d s \quad \forall v \in V_{D}(\Omega), \\
\int_{\Omega}\left(\sigma^{\prime}(u) \psi_{1} \nabla \varphi+\sigma(u) \nabla \psi_{2}\right) \cdot \nabla w d x=0 \quad \forall w \in H_{0}^{1}(\Omega) .
\end{gathered}
$$

Since the minimum of $J$ is achieved at $\beta^{*}$ and for small $\varepsilon>0, \beta^{*}+\varepsilon \ell \in U_{\mathcal{M}}$, we 
obtain

$$
\begin{aligned}
0 & \leq \lim _{\varepsilon \rightarrow 0^{+}} \frac{J\left(\beta^{*}+\varepsilon \ell\right)-J\left(\beta^{*}\right)}{\varepsilon} \\
& =\int_{\Omega} \psi_{1} d x+\int_{\Gamma_{R}} 2 \beta^{*} \ell d s=\int_{\Omega}\left(\begin{array}{ll}
\psi_{1} & \psi_{2}
\end{array}\right)\left(\begin{array}{c}
1 \\
0
\end{array}\right) d x+\int_{\Gamma_{R}} 2 \beta^{*} \ell d s \\
& =-\int_{\Omega} \nabla p \nabla \psi_{1} d x-\int_{\Gamma_{R}} \beta^{*} p \psi_{1} d s+\int_{\Omega} \psi_{1} \sigma^{\prime}(u)|\nabla \varphi|^{2} p d x-\int_{\Omega} \psi_{1} \sigma^{\prime}(u) \nabla \varphi \nabla q d x \\
& +2 \int_{\Omega} p \sigma(u) \nabla \varphi \cdot \nabla \psi_{2} d x-\int_{\Omega} \sigma(u) \nabla q \cdot \nabla \psi_{2} d x+\int_{\Gamma_{R}} 2 \beta^{*} \ell d s \\
& =\left\{-\int_{\Omega} \nabla p \nabla \psi_{1} d x-\int_{\Gamma_{R}} \beta^{*} p \psi_{1} d s+\int_{\Omega} \psi_{1} \sigma^{\prime}(u)|\nabla \varphi|^{2} p d x\right. \\
& \left.+2 \int_{\Omega} p \sigma(u) \nabla \varphi \nabla \psi_{2} d x\right\}+\left[-\int_{\Omega} \psi_{1} \sigma^{\prime}(u) \nabla \varphi \cdot \nabla q d x-\int_{\Omega} \sigma(u) \nabla q \cdot \nabla \psi_{2} d x\right] \\
& +\int_{\Gamma_{R}} 2 \beta^{*} \ell d s=\int_{\Gamma_{R}} \ell\left(u-u_{1}\right) p d s+\int_{\Gamma_{R}} 2 \beta^{*} \ell d s,
\end{aligned}
$$

where we integrated by parts and used (5.25) with test functions $p$ and $q$. Hence,

$$
\int_{\Gamma_{R}} \ell\left(2 \beta^{*}+\left(u-u_{1}\right) p\right) d s \geq 0 .
$$

By the same argument as in 14] one can obtain the explicit characterization (5.24) of the optimal control. Namely,

(i) Taking the variation $\ell$ to have support on the set $\left\{x \in \Gamma_{R}: \lambda<\beta^{*}(x)<\mathcal{M}\right\}$ implies that the variation $\ell(x)$ can be of any sign, and therefore we obtain $2 \beta^{*}+\left(u-u_{1}\right) p=0$ which leads to $\beta^{*}=-\frac{\left(u-u_{1}\right) p}{2}$.

(ii) On the set $\left\{x \in \Gamma_{R}: \beta^{*}(x)=\mathcal{M}\right\}$, the variation must satisfy $\ell(x) \leq 0$ and therefore we get $2 \beta^{*}+\left(u-u_{1}\right) p \leq 0$ implying $\mathcal{M}=\beta^{*}(x) \leq-\frac{\left(u-u_{1}\right) p}{2}$.

(iii) On the set $\left\{x \in \Gamma_{R}: \beta^{*}(x)=\lambda\right\}$, the variation must satisfy $\ell(x) \geq 0$. This implies $2 \beta^{*}+\left(u-u_{1}\right) p \leq 0$ and thus $\lambda=\beta^{*}(x) \geq-\frac{\left(u-u_{1}\right) p}{2}$.

Combining cases (i), (ii), and (iii) gives the explicit characterization (5.24) of the optimal control $\beta$.

Substituting (5.24) into the state system (1.1) and the adjoint equations (5.23) we obtain the optimality system:

$$
\begin{aligned}
\Delta u^{*}+\sigma\left(u^{*}\right)\left|\nabla \varphi^{*}\right|^{2} & =0 \text { in } \Omega, \\
\nabla \cdot\left(\sigma\left(u^{*}\right) \nabla \varphi^{*}\right) & =0 \text { in } \Omega, \\
\Delta p+\sigma^{\prime}\left(u^{*}\right)\left|\nabla \varphi^{*}\right|^{2} p-\sigma^{\prime}\left(u^{*}\right) \nabla \varphi^{*} \cdot \nabla q & =1 \text { in } \Omega, \\
\nabla \cdot\left[-2 p \sigma\left(u^{*}\right) \nabla \varphi^{*}+\sigma\left(u^{*}\right) \nabla q\right] & =0 \text { in } \Omega, \\
\frac{\partial p}{\partial n}+\min \left(\max \left(-\left(u^{*}-u_{1}\right) p / 2,0\right), \mathcal{M}\right) p & =0 \text { on } \Gamma_{R}, \\
p & =0 \text { on } \Gamma_{D}, \\
q & =0 \text { on } \partial \Omega, \\
\frac{\partial u^{*}}{\partial n}+\min \left(\max \left(-\left(u^{*}-u_{1}\right) p / 2,0\right), \mathcal{M}\right)\left(u^{*}-u_{1}\right) & =0 \text { on } \Gamma_{R}, \\
u & =u_{0} \text { on } \Gamma_{D}, \\
\varphi^{*} & =\varphi_{0} \text { on } \partial \Omega .
\end{aligned}
$$


Note that existence of solution to the optimality system (5.27) follows from the existence of solution to the state system (1.1) and Theorem 5.2 .

\section{REFERENCES}

[1] M. R. Sidi Ammi And Delfim F. M. TorRes, Optimal control of nonlocal thermistor equations, Internat. J. Control, 85 (2012), pp. 1789-1801.

[2] S. N. AntontSEV AND M. Снipot, The thermistor problem: existence, smoothness, uniqueness, and blow up, SIAM J. Math. Anal., 25 (1994), pp. 1128-1156.

[3] K. AtKinson And W. Han, Theoretical numerical analysis. A functional analysis framework, Springer, New York, 2001.

[4] A. Bensoussan, J.-L. Lions, and G. Papanicolau, Asymptotic analysis for periodic structures, North Holland, Amsterdam, 1978.

[5] X. Chen, Existence and regularity of solutions of a nonlinear nonuniformly elliptic system arising from a thermistor problem, J. Part. Diff. Eq. 7 (1994), pp. 19-34.

[6] G. Cimatti, Existence of weak solutions for the nonstationary problem of the Joule heating of a conductor, Ann. Mat. Pura Appl. (4), 162 (1992), pp. 33-42.

[7] G. Cimatti and G. Prodi, Existence results for a nonlinear elliptic system modeling a temperature dependent electrical resistor, Ann. Mat. Pura Appl. (4), 63 (1989), pp. 227-236.

[8] G. CimatTi, A bound for the temperature in the thermistor's problem, IMA J. Appl. Math., 40 (1988), pp. 15-22.

[9] H. Diesselhorst, Ueber das problem eines electrisch erwärmten Leiters, Ann. Physik, 40 (1900), pp. 312-325.

[10] A. C. Fowler, I. FrigaArd, And S. D. Howison, Temperature surges in current-limiting circuit devices, SIAM J. Appl. Math., 52 (1992), pp. 998-1011.

[11] D. Gilbarg And N. S. Trudinger, Elliptic partial differential equations of second order, 2nd edition, Springer-Verlag, Berlin, 1983.

[12] D. Homberg, C. Meyer, J. Rehberg and W. Ring, Optimal control for the thermistor problem, SIAM J. Control Optim., 48 (2010), no. 5, pp. 3449-3481.

[13] S. D. Howison, J. F. Rodrigues, And M. Shillor, Stationary solutions to the thermistor problem, J. Math. Anal. Appl., 174 (1993), pp. 573-588.

[14] V. Hrynkiv, S. Lenhart, and V. Protopopescu, Optimal control of a convective boundary condition in a thermistor problem, SIAM J. Control Optim., 47 (2006), pp. 20-39.

[15] K. KwoK, Complete guide to semiconductor devices, McGraw-Hill, New York, 1995.

[16] H-C. Lee And T. Shilkin, Analysis of optimal control problem for the two-dimensional thermistor system, SIAM J. Control Optim., 44 (2005), pp. 268-282.

[17] E. D. Maclen, Thermistors, Electrochemical Publications, Glasgow, 1979.

[18] H. Meinlschmidt, C. Meyer, and J. Rehberg, Optimal control of the thermistor problem in three spatial dimensions, arXiv: 1603.04305v1, [math.OC], 14 Mar 2016.

[19] N. G. Meyers, An $L^{p}$ estimate for the gradient of solutions of second order elliptic divergence equations, Ann. Sc. Norm. Super. Pisa, 17 (1963), pp. 189-206.

[20] J. F. Rodrigues, Obstacle problems in mathematical physics, North - Holland Mathematics Studies, vol. 134, North-Holland, Amsterdam, 1987, 352 pp.

[21] P. Shi, M. Shillor, And X. Xu, Existence of a solution to the Stefan problem with Joule's heating, J. Differential Equations, 105 (1993), pp. 239-263.

[22] M. E. TAYLOR, Partial differential equations III. Nonlinear equations, Springer, New York, 1996.

[23] X. XU, The thermistor problem with conductivity vanishing for large temperature, Proc. Roy. Soc. Edinburgh, 124A (1994), pp. 1-21.

[24] X. Xu, Local regularity theorems for the stationary thermistor problem, Proc. Roy. Soc. Edinburgh, 134A (2004), pp. 773-782.

[25] X. Xu, Exponential integrability of temperature in the thermistor problem, Differential Integral Equations, 17 (2004), pp. 571-582.

[26] G. YuAn, Regularity of solutions of the nonstationary thermistor problem, Appl. Anal., 53 (1994), pp. 149-164

[27] E. ZeIDler, Nonlinear functional analysis and its applications IV, Springer, New York, 1988.

[28] S. Zhou and D. R. Westbrook, Numerical solution of the thermistor equations, J. Comput. Appl. Math., 79 (1997), pp. 101-118. 